\title{
Explicit mixed strain-displacement finite elements for compressible and quasi-incompressible elasticity and plasticity
}

\author{
M. Cervera, N. Lafontaine, R. Rossi and M. Chiumenti \\ International Center for Numerical Methods in Engineering (CIMNE) \\ Technical University of Catalonia (UPC) \\ Edificio C1, Campus Norte, Jordi Girona 1-3, 08034 Barcelona, Spain.
}

KEYWORDS: explicit mixed finite elements, stabilization, incompressibility, plasticity, strain softening, strain localization, mesh independence.

\begin{abstract}
This paper presents an explicit mixed finite element formulation to address compressible and quasi-incompressible problems in elasticity and plasticity. This implies that the numerical solution only involves diagonal systems of equations. The formulation uses independent and equal interpolation of displacements and strains, stabilized by variational subscales (VMS). A displacement sub-scale is introduced in order to stabilize the pressure field. Compared to standard irreducible formulation, the proposed formulation yields improved strain and stress fields. The paper investigates the effect of this enhacement in accuracy in problems involving strain softening and localization leading to failure, using low order finite elements with linear continuous strain and displacement fields ( $P 1 P 1$ triangles in $2 \mathrm{D}$ and tetrahedra in $3 \mathrm{D})$ in conjunction with associative frictional Mohr-Coulomb and Drucker-Prager plastic models. The performance of the strain/displacement formulation under compressible and nearly incompressible deformation patterns is assessed and compared to analytical solutions for plane stress and plane strain situations. Benchmark numerical examples show the capacity of the mixed formulation to predict correctly failure mechanisms with localized patterns of strain, virtually free from any dependence of the mesh directional bias. No auxiliary crack tracking technique is necessary.
\end{abstract}




\section{Introduction}

In computational solid mechanics it is common to use displacements as nodal unknowns and to calculate strains by differentiation of the interpolated displacement field. These so-call irreducible formulations are very effective in a wide range of engineering problems; however, in certain cases, they lead to unstable, locking or mesh dependent results.

For instance, the performance of standard low order irreducible finite elements is extremely poor in quasi-incompressible situations, both in elasticity and plasticity. This is due to volumetric locking that brings in overstiff behavior and pressure oscillations that hamstring the stress field. There exists considerable literature proposing solutions to this problem. Using displacements $\boldsymbol{u}$ as primary variables, the first proposals were based on reduced integration [30, 39], and extended to the use of assumed deformations [47] and the B-bar method [31]. Mixed pressure-displacement $\boldsymbol{u}-p$ approaches were introduced in the 90's and used thenceforth to address the incompressible limit $[17,29,38,41,49]$. The reason for using the pressure as independent variable is to gain control on it and ensure stability; this reflects in an overall satisfactory behavior of strains and stresses in quasi-incompressible situations.

Discrete irreducible formulations have also proved inadequate in strain localization induced by softening constitutive behavior. In this instance, mesh dependent solutions are obtained, with the bane of failure mechanisms, peak loads and energy dissipation being grossly mispredicted. Like incompressibility, failure mechanics has been an active topic of research in the last 30 years, with alternative explanations for the issue and consequent proposals being advocated, going from the in-

troduction of localization limiters, regularized and non-local constitutive models to the more recent phase-field simulation

(FE) formulations, fr

J2 plasticity with str

sults. In these works,

hence, the rate of convergence for the angular

tion. More recently, Cer
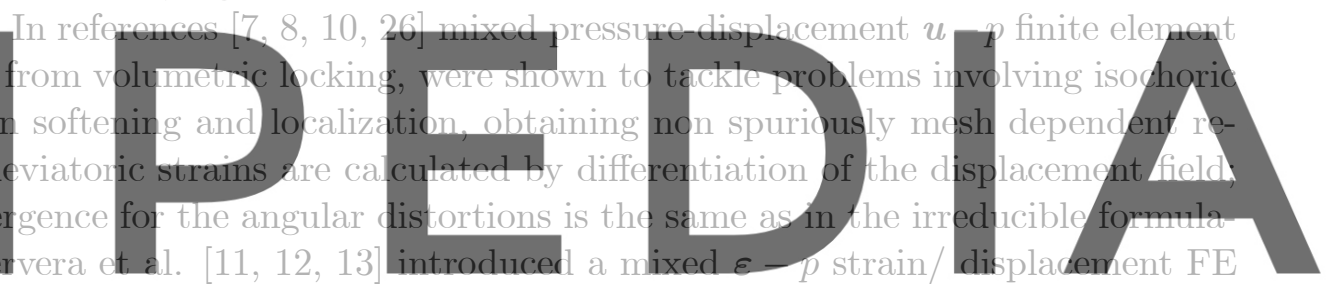

formulation and have applied it to address problems of strain localization using compressible and

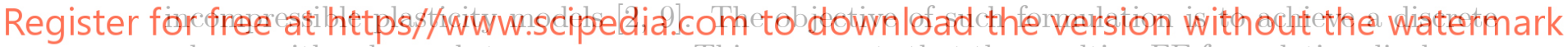
scheme with enhanced stress accuracy. This represents that the resulting FE formulation displays a global rate of convergence on stresses higher than the corresponding irreducible formulation. Such improvement of the convergence estimates also applies at local level. And this characteristic proves to be crucial in strain localization problems involving softening materials.

In the previously referenced mixed $\mathrm{FE}$ element formulations, linear interpolation spaces were used for all primary variables, displacements and pressure/strain. A three field formulation is also possible [16]. All these mixed schemes are stabilized via the Variational Multi-Scale method (VMS) [20, 31, 32, 33, 42], and, specifically, the Orthogonal Subgrid Scales method, in order to gain control of all the variables while circumventing the restrictiveness of the inf-sup compatibility conditions on the choice of the interpolation spaces. Similar mixed approaches have been proposed using alternative stabilization techniques, like SUPG [4, 5, 29, 36] or FIC [42].

The mixed displacement-strain formulation involves a larger number of degrees of freedom (dofs) per node than in the irreducible approach, which results in weightier linear systems to be solved. In reference [35], the authors proposed an explicit $\boldsymbol{\varepsilon}-\boldsymbol{u}$ strain/displacement mixed finite element 
formulation (MEX-FEM in the following) to address dynamic geometrically nonlinear problems in solid mechanics. It was shown there that combining the mixed formulation with an explicit time integration scheme yields a completely explicit mixed approach that it is very cost effective, as only diagonal systems of equations need to be solved.

Based on this, the objectives of the present work are three-fold: (i) to extend the applicability of MEX-FEM into the quasi-incompressible elastic regime, (ii) to extend the applicability of MEX-FEM into the compressible and quasi-incompressible plastic regime, (iii) to demonstrate that an explicit mixed formulation can satisfactorily solve quasi-static materially nonlinear problems involving strain localization.

Inelastic plastic flow is a directional phenomenon. Assuming associative plasticity, it occurs in the direction normal to the yield surface. An additional objective of this work is to investigate the performance of the proposed mixed formulation in strain localization situations to assess its satisfactory performance under directional inelastic behavior, without spurious stress locking and without the need of auxiliary discontinuity tracking procedures.

The organization of this paper is as follows. In section 2, the mixed approach is presented, including the subscale approach and the corresponding stabilization procedure. Specifically, a displacement subscale is introduced to tackle quasi-incompressible situations. Relevant aspects of the numerical implementation are recalled. Section 3 presents the extension of the formulation to the elasto-plastic range. The Mohr-Coulomb and Drucker-Prager plasticity models, selected as target models, are introduced. Constitutive integration, the return algorithm and some general aspects about the orientation of the localization band are given. Section 4 presents selected numerical examples involving unstructured and structured low order finite elements meshes (triangles in 2D

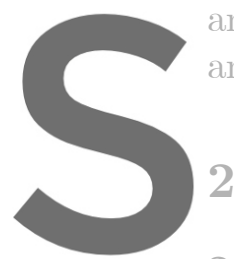
and tetrahedra in $3 \mathrm{D}$ and robustness of the

\section{2}

\subsection{Mixed strong and weak forms}
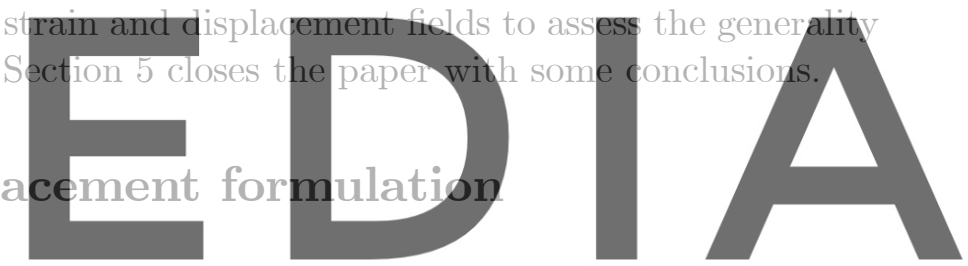

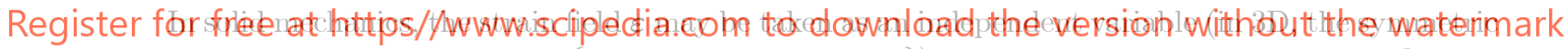
tensor $\varepsilon$ has six components, $\left.\left\{\varepsilon_{x x}, \varepsilon_{y y}, \varepsilon_{z z}, \varepsilon_{x y}, \varepsilon_{y z}, \varepsilon_{x z}\right\}\right)$, additionally to the displacement fields $u$ $\left(u=\left\{u_{x}, u_{y}, u_{z}\right\}\right.$, in 3D).

In this case, the strong form of the problem, at continuous level, can be written as: find the displacement field $\boldsymbol{u}$, its time derivatives (velocity $\dot{\boldsymbol{u}}$ and acceleration $\ddot{\boldsymbol{u}}$ ) and the strain field $\boldsymbol{\varepsilon}$, given the body forces $\boldsymbol{b}$, so that:

$$
\begin{aligned}
\nabla \cdot \boldsymbol{\sigma}+\boldsymbol{b} & =\rho \ddot{\boldsymbol{u}} \text { in } \Omega \\
\boldsymbol{\varepsilon}-\nabla^{s} \boldsymbol{u} & =\mathbf{0} \text { in } \Omega
\end{aligned}
$$

where $\Omega \subset \mathbb{R}^{n_{\text {dim }}}$ is the volume occupied by the solid in the space of $n_{\text {dim }}$ dimensions, $\rho$ is the material density and $\sigma$ is the stress tensor, assumed to be expressed in terms of the strains $\sigma=\sigma(\varepsilon)$, via a non-linear constitutive relationship of the type $\boldsymbol{\sigma}=\boldsymbol{C}: \varepsilon$, with $\boldsymbol{C}=\boldsymbol{C}(\varepsilon)$ being the (secant) nonlinear constitutive tensor; see Section 3 for the application to plasticity models. Eq. (1) represents the balance of momentum and Eq. (2) is the kinematic equation. 
Eqs. (1) and (2) must be satisfied for all times $t \in[0, T]$ of the time interval of interest. Additionally, the problem is subjected to appropriate boundary conditions, Dirichlet and Neumann conditions applied respectively in parts $\partial \Omega_{u}$ and $\partial \Omega_{t}$ of the boundary $\partial \Omega$, so that $\partial \Omega=\partial \Omega_{u} \cup \partial \Omega_{t}$ and $\partial \Omega_{u} \cap \partial \Omega_{t}=\varnothing$. Also, the variables $\boldsymbol{\varepsilon}, \boldsymbol{u}$ and the derivative $\dot{\boldsymbol{u}}$ are subjected to initial conditions at $t=t_{0}$, so that, $\left.\boldsymbol{\varepsilon}\right|_{t=t_{0}}=\varepsilon_{0}=\nabla^{s} \boldsymbol{u}_{0},\left.\boldsymbol{u}\right|_{t=t_{0}}=\boldsymbol{u}_{0}$ and $\left.\dot{\boldsymbol{u}}\right|_{t=t_{0}}=\boldsymbol{v}_{0}$.

Multiplying Eqs. (1) and (2) by the respective test functions and integrating by parts Eq. (1), the weak form of the problem reads:

$$
\begin{array}{rlrl}
\int_{\Omega} \nabla^{s} \boldsymbol{\omega}: \boldsymbol{\sigma} d \Omega+\int_{\Omega} \boldsymbol{\omega} \cdot \rho \ddot{\boldsymbol{u}} d \Omega & =\int_{\partial \Omega_{t}} \boldsymbol{\omega} \cdot \overline{\boldsymbol{t}} d \Gamma+\int_{\Omega} \boldsymbol{\omega} \cdot \boldsymbol{b} d \Omega & & \forall \boldsymbol{\omega} \\
\int_{\Omega} \boldsymbol{\gamma} \cdot \varepsilon d \Omega & =\int_{\Omega} \boldsymbol{\gamma} \cdot \nabla^{s} \boldsymbol{u} d \Omega & \forall \boldsymbol{\gamma}
\end{array}
$$

where $\boldsymbol{\omega} \in \mathcal{V}$ and $\gamma \in \mathcal{T}$ are the test functions for the displacement and strain fields, respectively; $\mathcal{V}$ and $\mathcal{T}$ are the functional spaces for the admissible displacement and strain, fields; $\overline{\boldsymbol{t}}=\boldsymbol{\sigma} \boldsymbol{n}$ are the tractions on the outward direction $n$ in $\partial \Omega_{t}$.

To obtain the discrete form of the problem, a discrete displacement field $u_{h}$, its discrete time derivatives $\left(\dot{\boldsymbol{u}}_{h}\right.$ and $\left.\ddot{\boldsymbol{u}}_{h}\right)$ and a discrete strain field $\varepsilon_{h}$ are defined. The discrete counterpart of Eqs. (3) and (4) is then:

$$
\int_{\Omega} \nabla^{s} \omega_{h}: \sigma_{h} d \Omega+\int_{\Omega} \omega_{h} \cdot \rho \ddot{u}_{h} d \Omega=\int_{\partial \Omega_{t}} \omega_{h} \cdot \bar{t} d \Gamma+\int_{\Omega} \omega_{h} \cdot b d \Omega \quad \forall \omega_{h}
$$
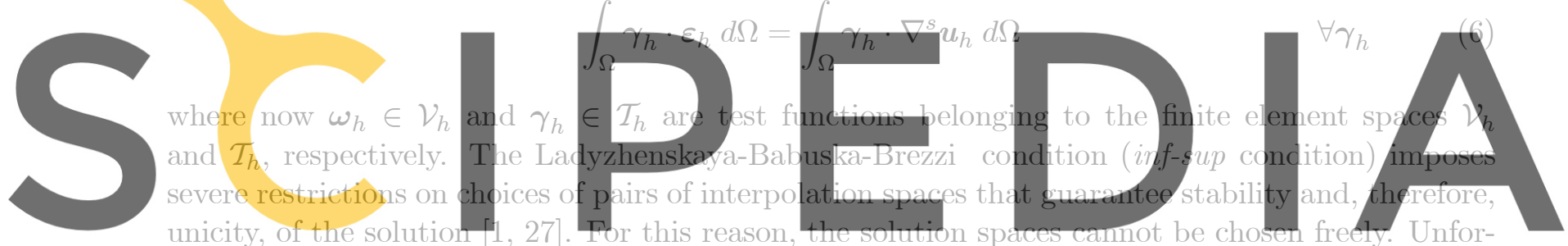
unicity, of the solution $[1,27]$. For this reason

tunately, most of low order mixed finite elements, including the simple P1P1 element, with equal

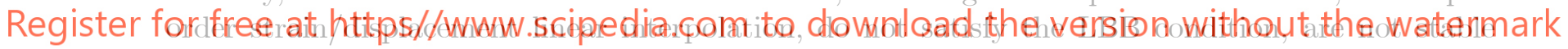

and present spurious oscillations in the displacement field that jeopardize the numerical solution.

Conveniently, the restrictions posed by the LBB condition may be circumvented by means of stabilization techniques to provide the necessary stability to the discrete mixed variational form. In these stabilized finite element forms, some appropriately constructed terms are added to Eqs. (5) and (6); these terms are residual-based and vanish on mesh refinement, so that the consistency of the formulation is not affected. More details are given in the next Section

Additionally, as Eqs. (5) and (6) are discrete in space but still continuous in time, a time discretization algorithm is required. In this work, an explicit central differences scheme is used.

\subsection{Variational Multiscale Stabilization}

The stabilization method used in this work belongs to the remarkably successful framework of Variational Multiscale method (VMS) of Hughes and collaborators [33]. The fundamental idea of the method is to distinguish two scales or resolution levels, a coarse scale, at mesh level, that can 
be solved by the finite element approximation and another, finer one, that cannot be captured by the mesh resolution, and that is called subscale. Accordingly, the approximation of the continuous strain and displacement fields must contain components from both the scale and the subscale:

$$
\begin{aligned}
\varepsilon & =\varepsilon_{h}+\widetilde{\varepsilon} \\
\boldsymbol{u} & =\boldsymbol{u}_{h}+\widetilde{\boldsymbol{u}}
\end{aligned}
$$

where $\varepsilon_{h} \in \mathcal{T}_{h}$ and $\boldsymbol{u}_{h} \in \mathcal{V}_{h}$ are fields defined in the coarse finite element scale and $\widetilde{\boldsymbol{\varepsilon}} \in \widetilde{\mathcal{T}}$ and $\widetilde{\boldsymbol{u}} \in \tilde{\mathcal{V}}$ are the corresponding subgrid scales. Subsequently, the velocities are approximated as $\dot{\boldsymbol{u}}=\dot{\boldsymbol{u}}_{h}+\dot{\widetilde{\boldsymbol{u}}}$ and the accelerations as $\ddot{\boldsymbol{u}}=\ddot{\boldsymbol{u}}_{h}+\ddot{\widetilde{\boldsymbol{u}}}$.

Introducing the test functions $\widetilde{\boldsymbol{\omega}} \in \widetilde{\mathcal{V}}$ and $\widetilde{\gamma} \in \widetilde{\mathcal{T}}$ in the corresponding subscale spaces, the interpolation spaces are now extended to $\mathcal{T} \simeq \mathcal{T}_{h} \oplus \widetilde{\mathcal{T}}$ and $\mathcal{V} \simeq \mathcal{V}_{h} \oplus \widetilde{\mathcal{V}}$, respectively. Each particular stabilized finite element method is characterized by its choice of the spaces $\widetilde{\mathcal{V}}$ and $\widetilde{\mathcal{T}}$. The classical Galerkin method is recovered for $\widetilde{\mathcal{V}}=\widetilde{\mathcal{T}}=\{0\}$.

Approximating $\boldsymbol{C}=\boldsymbol{C}(\varepsilon) \simeq \boldsymbol{C}\left(\varepsilon_{h}\right)([11,12])$, and also $\dot{\varepsilon}_{p}=\dot{\varepsilon}_{p}(\boldsymbol{\sigma}) \simeq \dot{\varepsilon}_{\boldsymbol{p}}\left(\boldsymbol{\sigma}_{h}\right)$, the stress tensor in Eq. (43) can be written as:

$$
\sigma(\varepsilon)=C\left(\varepsilon_{h}\right): \varepsilon_{h}+C\left(\varepsilon_{h}\right): \widetilde{\varepsilon}=\sigma_{h}+\widetilde{\sigma}
$$

These approximations imply that the subscale is a perturbation of the grid scale. In practice, they imply that the constitutive model is assumed to be driven by the grid scale.

Introducing the two-scale approximation (Eqs. (7), (8) and (9) and their counterparts for the test functions) into Eqs. (3) and (4), each one of these unfold into two equations, one corresponding to each scale. The equation of balance of momentum equ
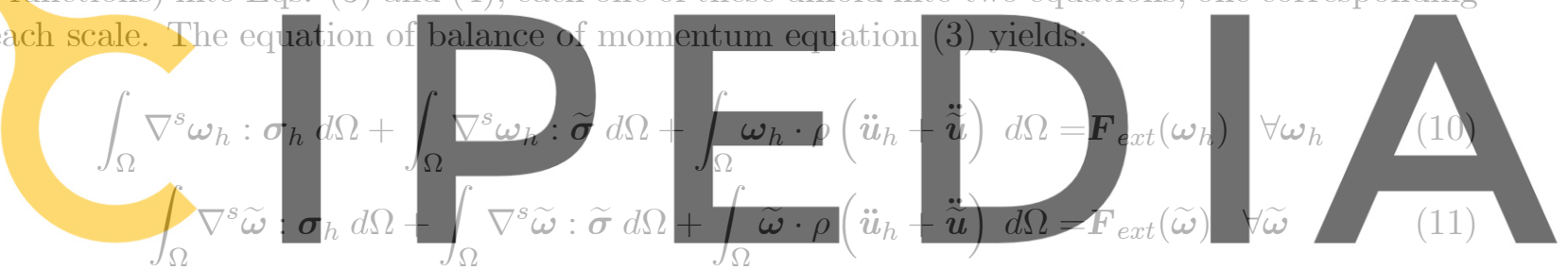

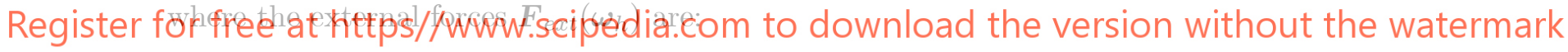

$$
\mathbb{F}_{\text {ext }}\left(\omega_{h}\right)=\int_{\partial \Omega} \omega_{h} \cdot \bar{t} d \Gamma+\int_{\Omega} \omega_{h} \cdot b d \Omega
$$

Similarly, the kinematic equation (4) yields:

$$
\begin{gathered}
\int_{\Omega} \gamma_{h} \cdot \varepsilon_{h} d \Omega+\int_{\Omega} \gamma_{h} \cdot \widetilde{\varepsilon} d \Omega=\int_{\Omega} \gamma_{h} \cdot \nabla^{s} \boldsymbol{u}_{h} d \Omega-\int_{\Omega}\left(\nabla \cdot \gamma_{h}\right) \cdot \widetilde{\boldsymbol{u}} d \Omega \quad \forall \gamma_{\boldsymbol{h}} \\
\int_{\Omega} \widetilde{\gamma} \cdot \varepsilon_{h} d \Omega+\int_{\Omega} \widetilde{\gamma} \cdot \widetilde{\varepsilon} d \Omega=\int_{\Omega} \widetilde{\gamma} \cdot \nabla^{s} \boldsymbol{u}_{h} d \Omega+\int_{\Omega} \widetilde{\gamma} \cdot \nabla^{s} \widetilde{\boldsymbol{u}} d \Omega \quad \forall \widetilde{\gamma}
\end{gathered}
$$

where integration by parts has been used in Eq. (13) and the resulting integral over the boundary has been neglected $[11,20]$.

Equations (11) and (14) cannot be solved in the finite element space, and so an approximation for the subscales is required. Note that their point-wise values are not needed, as only the stabilizing 
integral terms in (10) and (13) are called for [11]. This is why the stabilization method is termed variational multiscale method.

The residuals corresponding to the finite element scale are:

$$
\begin{aligned}
\boldsymbol{r}_{u, h} & =\rho \ddot{\boldsymbol{u}}_{h}-\nabla \cdot \boldsymbol{\sigma}_{h}-\boldsymbol{b} \\
\boldsymbol{r}_{\varepsilon, h} & =\nabla^{s} \boldsymbol{u}_{h}-\boldsymbol{\varepsilon}_{h}
\end{aligned}
$$

and Eqs. (11) and (14) can be written in terms of these as:

$$
\begin{aligned}
\int_{\Omega} \widetilde{\boldsymbol{\omega}} \cdot(\nabla \cdot(\boldsymbol{C}: \widetilde{\boldsymbol{\varepsilon}})) d \Omega-\int_{\Omega} \rho \widetilde{\boldsymbol{\omega}} \cdot \ddot{\widetilde{\boldsymbol{u}}} d \Omega & =\int_{\Omega} \widetilde{\boldsymbol{\omega}} \cdot \boldsymbol{r}_{u, h} d \Omega \\
\int_{\Omega} \widetilde{\boldsymbol{\gamma}} \cdot \widetilde{\boldsymbol{\varepsilon}} d \Omega-\int_{\Omega} \widetilde{\boldsymbol{\gamma}} \cdot \nabla^{s} \widetilde{\boldsymbol{u}} d \Omega & =\int_{\Omega} \widetilde{\boldsymbol{\gamma}} \cdot \boldsymbol{r}_{\varepsilon, h} d \Omega
\end{aligned}
$$

which clearly relate the subscales $\widetilde{\varepsilon}$ and $\widetilde{\boldsymbol{u}}$ with the projections of the residuals from the finite element scale, $\boldsymbol{r}_{\varepsilon, h}$ and $\boldsymbol{r}_{u, h}$, onto the subscale spaces, $\widetilde{\mathcal{T}}$ and $\widetilde{\mathcal{V}}$, respectively.

The second time derivative of the displacement subscale $\widetilde{u}$ appears in Eq. (17), so it is necessary to integrate this in time [23]. To this end, let us consider a uniform partition of the time interval $[0, T]$ of size $\Delta t$, so that time $t^{n}=n \Delta t$. Using a central difference scheme, the second time derivative is approximated as:

$$
\ddot{u}^{n}=\frac{1}{\Delta t^{2}}\left(\widetilde{u}^{n+1}-2 \widetilde{u}^{n}+\widetilde{u}^{n-1}\right)
$$

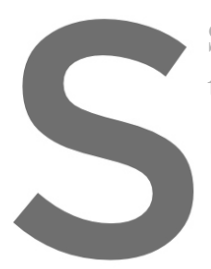

Substituting (19) int

tively, Eqs. (17) and $[20,23]$ :
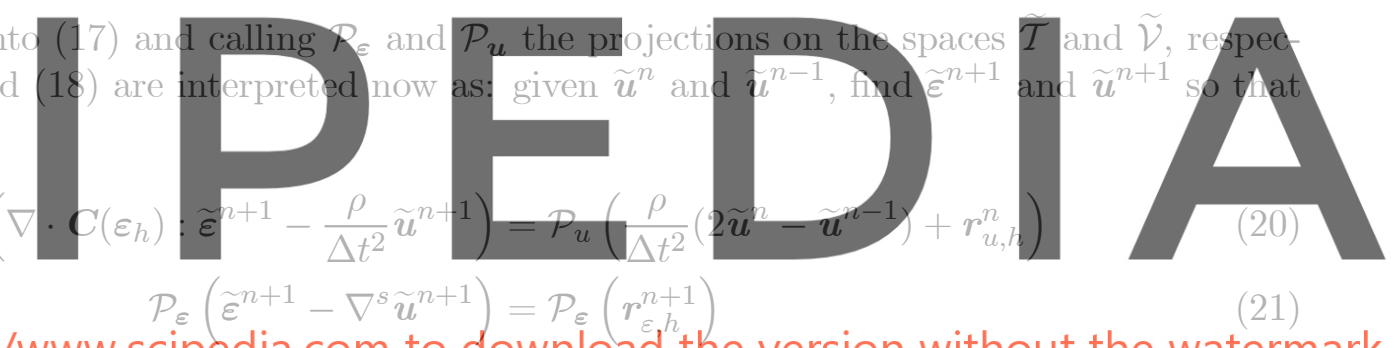

(21)

Register for free at https//www.scipedia.com to download the version without the watermark

Following the arguments in reference [22], $\widetilde{\varepsilon}^{n+1}$ and $\widetilde{u}^{n+1}$ can be approximated within each element domain $\Omega_{e}$ as:

$$
\begin{aligned}
\widetilde{\boldsymbol{\varepsilon}}^{n+1} & =\tau_{\varepsilon} \mathcal{P}_{\varepsilon}\left(\boldsymbol{r}_{\varepsilon, h}^{n+1}\right) \\
\widetilde{\boldsymbol{u}}^{n+1} & =\tau_{u} \frac{\rho}{\Delta t^{2}}\left(2 \widetilde{\boldsymbol{u}}^{n}-\widetilde{\boldsymbol{u}}^{n-1}\right)+\tau_{u} \mathcal{P}_{u}\left(-\boldsymbol{r}_{u, h}^{n}\right)
\end{aligned}
$$

where $\tau_{u}$ and $\tau_{\varepsilon}$ are the stabilization parameters, given by

$$
\tau_{\varepsilon}=c_{\varepsilon} \frac{h}{L_{0}} \frac{\mu}{\mu_{0}} \text { and } \tau_{u}=\left(\frac{\rho}{\Delta t^{2}}+c_{u} \frac{\mu}{h L_{0}}\right)^{-1}
$$

where $c_{\varepsilon}>0$ and $c_{u}>0$ are non-dimensional algorithmic constants, $h$ is the element size, $L_{0}$ is a characteristic length of the problem and $\mu_{0}, \mu$ are the elastic and secant shear moduli. Numerical evidence shows that $c_{\varepsilon}$ and $c_{u}$ can be chosen in the range $[0.01,1.0]$. 


\subsection{Orthogonal Subscale Stabilization}

Is is clear from Eqs. (22) and (23) that there exist multiple possibilities to approximate the subscales, as they could be chosen in any space complementary to the finite element space. To fully define the stabilization method and compute the subscales, projections $\mathcal{P}_{\boldsymbol{\varepsilon}}$ and $\mathcal{P}_{\boldsymbol{u}}$ must be selected. The simplest possible projection is the identity $\mathcal{I}$. The procedure so defined is called Algebraic Sub-Scales (ASGS) [19]. Residual based stabilization techniques such as ASGS do not introduce any consistency error, as the exact solution annuls the added terms, so that the stabilized model converges to the solution of the problem in continuous format. Also, if designed properly, the convergence rate is not altered; that is, the subscale terms are appropriately dependent on the mesh size.

Alternatively, Codina proposed to take the subscale spaces $\widetilde{\mathcal{V}}$ and $\widetilde{\mathcal{T}}$ orthogonal to the finite element spaces $\mathcal{V}_{h}$ and $\mathcal{T}_{h}$. This is called the Orthogonal Sub-Scales (OSS) method [19, 20, 21]. This means that the strain and displacement spaces are approximated as $\mathcal{T} \simeq \mathcal{T}_{h} \oplus \mathcal{T}_{h}^{\perp}$ and $\mathcal{V} \simeq \mathcal{V}_{h} \oplus \mathcal{V}_{h}^{\perp}$, respectively. Constructing the subscales in the subspace orthogonal to the finite element subspace has several advantages over the many other possibilities. The main one is that it guarantees minimal numerical dissipation on the discrete solution, because it adds nothing to those components of the residual already belonging to the FE subspace. This maximizes accuracy for a given mesh. Additionally, in transient problems, the term corresponding to the time derivative belongs to the finite element space, and therefore, its orthogonal projection is null. This means that the mass matrix remains unaltered by the stabilization method, maintaining its structure and symmetry. In this work we will make use of this method.

\section{Let the orthogonal projection operator be $\mathcal{P}_{h}^{\perp}(\bullet)=(\bullet)-\mathcal{P}_{h}(\bullet)$, where $\mathcal{P}_{h}(\bullet)$ is the $L^{2}$ projection}

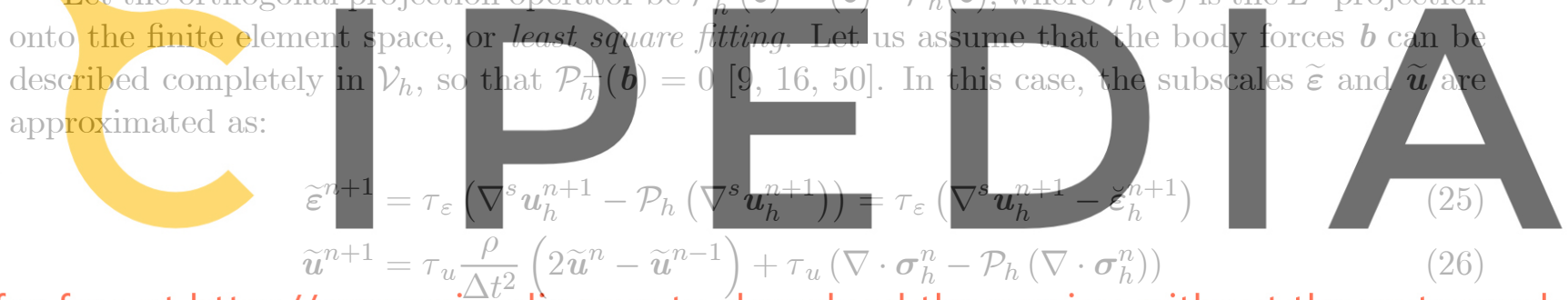

Register for free at https//www.scipedia.com to download the version without the watermark being $\breve{\varepsilon}_{h}^{n+1}=\mathcal{P}_{h}\left(\nabla^{s} u_{h}^{n+1}\right)$.

In quasi-incompressible situations it has been found to be effective to use the following alternative approximation for the displacement subscale:

$$
\widetilde{\boldsymbol{u}}^{n+1}=\tau_{u} \frac{\rho}{\Delta t^{2}}\left(2 \widetilde{\boldsymbol{u}}^{n}-\widetilde{\boldsymbol{u}}^{n-1}\right)+\tau_{u}\left(\nabla p_{h}^{n}-\mathcal{P}_{h}\left(\nabla p_{h}^{n}\right)\right)
$$

Here, the residual in Eq. (15) has been split as $\nabla \cdot \boldsymbol{\sigma}_{h}=\nabla p_{h}+\nabla \cdot \boldsymbol{S}_{h}$, being $\boldsymbol{S}_{h}$ the deviatoric stress tensor, and only the pressure gradient $\nabla p_{h}$ has been considered in the displacement subscale to ensure stability of the pressure field [9]. This has three advantages: (i) it reduces the computational stencil, (ii) it allows more selective norms to be defined for stability control and (iii) it has proved advantageous in problems involving singular or quasi-singular points. This variant of split-OSS belongs to the family of term-by-term stabilization methods; the introduced consistency error is of optimal order and the final convergence rate of the scheme is not altered. 


\subsection{Explicit stabilized mixed form}

To obtain the explicit stabilized form of the problem defined by the equations to be solved in the finite element space, Eqs. (10) and (13), the former needs to be discretized in time. Doing this via an explicit time advancing scheme is a well-known procedure for the irreducible formulation. Reference [35] discusses the relative merits of doing this for the mixed formulation, that is, incorporating the solution of the weak form of the geometric equation in the time marching scheme. The resulting explicit mixed method is conditionally stable, but, compared with its irreducible counterpart: (i) it shows enhanced strain and stress accuracy and (ii) it does not require a reduced critical time step.

On the one hand, the balance of momentum Eq. (10) is recalled at time $t^{n}$ as:

$$
\int_{\Omega} \nabla^{s} \boldsymbol{\omega}_{h}: \boldsymbol{C}\left(\varepsilon_{h}^{n}\right):\left[\varepsilon_{h}^{n}+\tau_{\varepsilon}\left(\nabla^{s} \boldsymbol{u}_{h}^{n}-\breve{\varepsilon}_{h}^{n}\right)\right] d \Omega+\int_{\Omega} \boldsymbol{\omega}_{h} \cdot \rho \ddot{\boldsymbol{u}}_{h}^{n} d \Omega=\boldsymbol{F}_{e x t}^{n}\left(\boldsymbol{\omega}_{h}\right) \quad \forall \boldsymbol{\omega}_{h}
$$

where the stress and strain subgrid scales from Eqs. (9) and (25) and the orthogonality condition $\int_{\Omega} \boldsymbol{\omega}_{h} \cdot \ddot{\widetilde{u}}^{n} d \Omega=\mathbf{0}$ have been used. On the other hand, the geometric Eq. (13) is recalled at time $t^{n+1}$ as:

$$
\int_{\Omega} \gamma_{h} \cdot \varepsilon_{h}^{n+1} d \Omega=\int_{\Omega} \gamma_{h} \cdot \nabla^{s} u_{h}^{n+1} d \Omega-\int_{\Omega}\left(\nabla \cdot \gamma_{h}\right) \cdot \widetilde{u}^{n+1} d \Omega \quad \forall \gamma_{h}
$$

where now the orthogonality condition $\int_{\Omega} \gamma_{h} \cdot \widetilde{\varepsilon} d \Omega=0$ has been used. From this, the strains $\varepsilon_{h}^{n+1}$ are obtained as:

$$
\varepsilon_{h}^{n+1}=\mathcal{P}_{h}\left(\nabla^{s} u_{h}^{n+1}-\left(\nabla \cdot \gamma_{h}\right) \cdot \widetilde{u}^{n+1}\right)=\breve{\varepsilon}_{h}^{n+1}-\mathcal{P}_{h}\left(\left(\nabla \cdot \gamma_{h}\right) \cdot \widetilde{u}^{n+1}\right)
$$
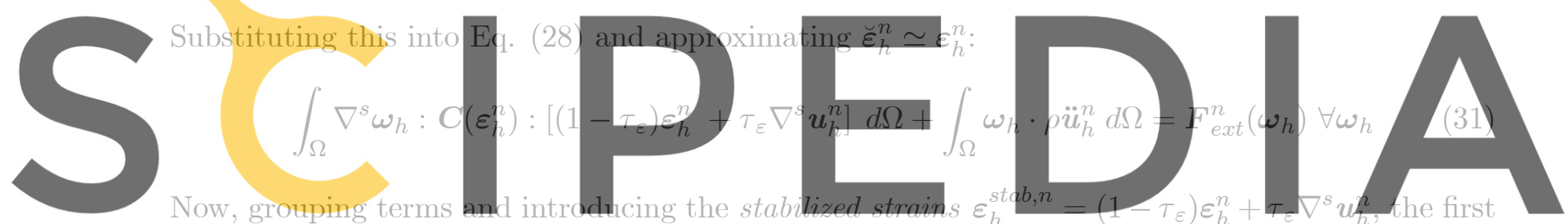

term in Eq. (31) is identified as the internal forces:

Register for free at https//www.scipedia.com to download the version without the watermark

$$
\mathbb{F}_{\text {int }}^{n}\left(\omega_{h}\right)=\int_{\Omega} \nabla^{s} \omega_{h}: \sigma^{n} d \Omega=\int_{\Omega} \nabla^{s} \omega_{h}: C\left(\varepsilon_{h}^{n}\right): \varepsilon_{h}^{\text {stab,n }} d \Omega
$$

This allows writing the equation of dynamic equilibrium at time $t^{n}$ in the usual matrix form:

$$
\boldsymbol{M} \ddot{\boldsymbol{U}}^{n}=\boldsymbol{F}_{\text {ext }}^{n}-\boldsymbol{F}_{\text {int }}^{n}
$$

where $\boldsymbol{U}^{n}$ is the vector of nodal displacements and $\boldsymbol{M}$ is the mass matrix, so that $\boldsymbol{F}_{\text {mass }}^{n}=\boldsymbol{M} \ddot{\boldsymbol{U}}^{n}=$ $\int_{\Omega} \boldsymbol{\omega}_{h} \cdot \rho \ddot{\boldsymbol{u}}_{h}^{n} d \Omega$ are the inertial forces. Introducing the central finite difference scheme to discretize the second time derivative as:

$$
\ddot{\boldsymbol{U}}^{n}=\frac{1}{\Delta t^{2}}\left(\boldsymbol{U}^{n+1}-2 \boldsymbol{U}^{n}+\boldsymbol{U}^{n-1}\right)
$$

the nodal displacements are obtained as:

$$
\boldsymbol{U}^{n+1}=\left(2 \boldsymbol{U}^{n}-\boldsymbol{U}^{n-1}\right)+\Delta t^{2} \boldsymbol{M}^{-1}\left(\boldsymbol{F}_{\text {ext }}^{n}-\boldsymbol{F}_{\text {int }}^{n}\right)
$$


This completes the definition of the explicit time marching procedure to solve the stabilized mixed strain/displacement problem. For each time step $t^{n+1}$ :

1. Evaluate the internal forces in previous time step $\boldsymbol{F}_{\text {int }}^{n}$ using Eq. (32).

2. Evaluate nodal displacements $\boldsymbol{U}^{n+1}$ using Eq. (35).

3. Evaluate the displacement subscale $\widetilde{\boldsymbol{u}}^{n+1}$ using Eq. (26).

4. Evaluate strains $\varepsilon_{h}^{n+1}$ using Eq. (30).

5. Go to next step.

The procedure is analogous to the one used for the standard irreducible formulation, apart from the additional steps 3 and 4. Therefore, it can be easily implemented in any FE code. Furthermore, the additional steps only require operations at element and node level that can be performed in parallel.

The scheme requires the solution of two systems of equations: the one in step 2 to obtain $U^{n+1}$ and another one in step 4 to perform the projection in Eq. (30). Both procedures are inexpensive, as the corresponding system matrices are constructed as diagonal.

\subsection{Damping}

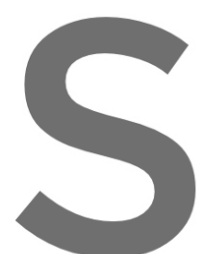

Viscous forces, that

computational solid mechanisms that dani

quasi-static cases, algor

to reach a steady-state

to filter out spurious high
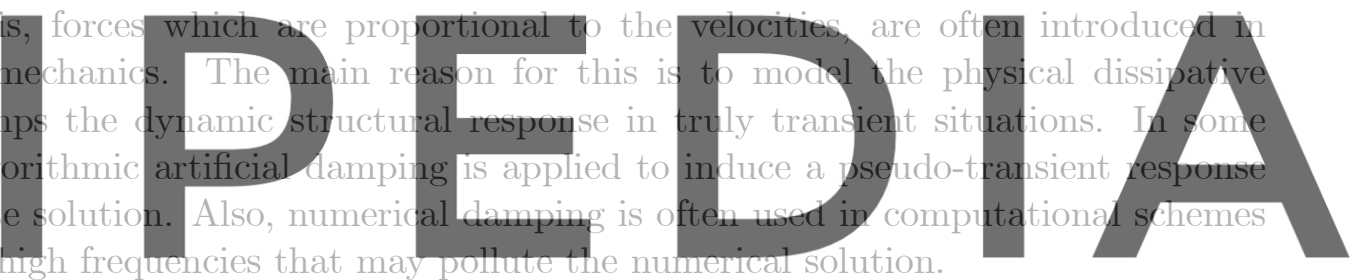

In the finite element grid scale, these forces are added to the equation of dynamic equilibrium

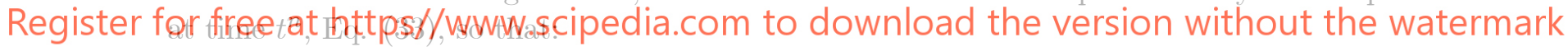

$$
M \ddot{U}^{n}+D \dot{U}^{n}=F_{e x t}^{n}-F_{i n t}^{n}
$$

where $\boldsymbol{F}_{v i s c}^{n}=\boldsymbol{D} \dot{\boldsymbol{U}}^{n}$ are the viscous forces, $\boldsymbol{D}$ being the damping matrix.

Making $\dot{\boldsymbol{U}}^{n}=\left(\boldsymbol{U}^{n}-\boldsymbol{U}^{n-1}\right) / \Delta t$, the nodal displacements at $t^{n+1}$ are obtained as:

$$
\boldsymbol{U}^{n+1}=\boldsymbol{M}^{-1}\left[[2 \boldsymbol{M}-\Delta t \boldsymbol{D}] \boldsymbol{U}^{n}-[\boldsymbol{M}-\Delta t \boldsymbol{D}] \boldsymbol{U}^{n-1}+\Delta t^{2}\left(\boldsymbol{F}_{e x t}^{n}-\boldsymbol{F}_{i n t}^{n}\right)\right]
$$

Mass- and stiffness-proportional damping, normally referred to as Rayleigh's damping, is commonly used in linear and nonlinear solid dynamic analysis, because of its computational benefits. In Rayleigh's damping, the damping matrix is assumed to be proportional to the mass and stiffness matrices:

$$
\boldsymbol{D}=\alpha \boldsymbol{M}+\beta \boldsymbol{K}
$$


where $\alpha$ and $\beta$ are damping coefficients. In order to preserve the efficiency of the explicit scheme, Rayleigh's damping is introduced in the form:

$$
\boldsymbol{U}^{n+1}=\left[(2-\alpha \Delta t) \boldsymbol{U}^{n}-(1-\alpha \Delta t) \boldsymbol{U}^{n-1}+\Delta t^{2} \boldsymbol{M}^{-1}\left(\boldsymbol{F}_{\text {ext }}^{n}-\boldsymbol{F}_{\text {int }}^{n}-\beta \boldsymbol{K} \dot{\boldsymbol{U}}^{n}\right)\right]
$$

where the viscous force term $\beta \mathbf{K} \dot{\boldsymbol{U}}^{n}$ can be evaluated element-by-element, without assembling a global stiffness matrix.

In the variational multiscale approach, mass proportional damping is also considered in the displacement subscale; using the time derivative $\dot{\overrightarrow{\boldsymbol{u}}}^{n}=\left(\widetilde{\boldsymbol{u}}^{n}-\widetilde{\boldsymbol{u}}^{n-1}\right) / \Delta t$, leads to a modified Eq. (26) for the evolution of the subscale:

$$
\widetilde{\boldsymbol{u}}^{n+1}=\tau_{u} \frac{\rho}{\Delta t^{2}}\left((2-\alpha \Delta t) \widetilde{\boldsymbol{u}}^{n}-(1-\alpha \Delta t) \widetilde{\boldsymbol{u}}^{n-1}\right)+\tau_{u}\left(\nabla p_{h}^{n}-\mathcal{P}_{h}\left(\nabla p_{h}^{n}\right)\right)
$$

\subsection{Computational implementation}

Explicit time integration is effective if the mass $\boldsymbol{M}$ in Eq. (35) is diagonal [18]. In this case, the computation of the nodal displacements in step 2 of the explicit scheme is trivial. The same applies to the projection matrix that appears to perform step 4. This is a mass-like matrix that may be constructed as purely diagonal. In this work, a close Gauss-Lobatto quadrature and mass proportional damping are used to ensure that all the matrices computed are diagonal.

Consider a $P 1 P 1$ 4-noded tetrahedal element with the same linear interpolation functions for strains and displacements. In the mixed formulation, strains are linear, and a 4-point quadrature rule, sampling at the element nodes is necessary to evaluate the internal forces.

same close quadrature $\Omega_{e}$.

The test functions interpolation functions Displacements and strai
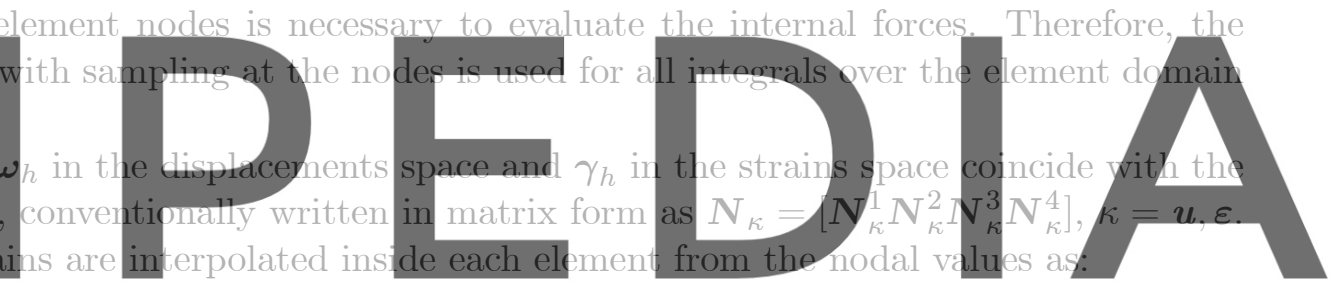

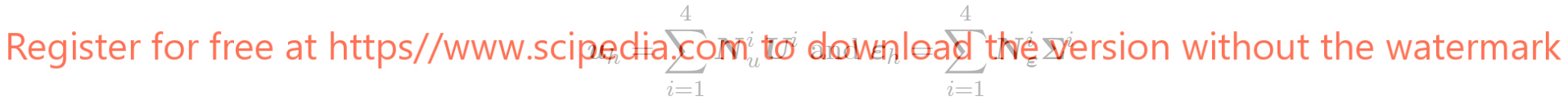

being $\boldsymbol{N}_{u}^{i}=\operatorname{diag}\left\{N_{i}, N_{i}, N_{i}\right\}$ the $3 \times 3$ diagonal submatrix for the displacement interpolation function, $\boldsymbol{U}^{i}=\left\{u_{x}^{i}, u_{y}^{i}, u_{z}^{i}\right\}$ the nodal displacements, $\boldsymbol{N}_{\varepsilon}^{i}=\operatorname{diag}\left\{N_{i}, N_{i}, N_{i}, N_{i}, N_{i}, N_{i}\right\}$ the $6 \times 6$ diagonal submatrix for the strain interpolation function, $\boldsymbol{\Sigma}^{i}=\left\{\varepsilon_{x x, h}^{i}, \varepsilon_{y y, h}^{i}, \varepsilon_{z z, h}^{i}, \varepsilon_{x y, h}^{i}, \varepsilon_{x z, h}^{i}, \varepsilon_{y z, h}^{i}\right\}$ the nodal strains, all at node $i$.

Note also that the tensor $\nabla^{s} \boldsymbol{\omega}_{h}$ appearing, for instance in Eq. (32), is the discrete symmetric gradient operator, usually noted as $\boldsymbol{B}_{u}$ in matrix form, with $\boldsymbol{B}_{u}=\left[\boldsymbol{B}_{1} \boldsymbol{B}_{2} \boldsymbol{B}_{3} \boldsymbol{B}_{4}\right]$, where each submatrix $\boldsymbol{B}_{i}$ is:

$$
\boldsymbol{B}_{i}=\left(\begin{array}{cccccc}
\frac{\partial N_{i}}{\partial X} & 0 & 0 & \frac{\partial N_{i}}{\partial Y} & \frac{\partial N_{i}}{\partial Z} & 0 \\
0 & \frac{\partial N_{i}}{\partial Y} & 0 & \frac{\partial N_{i}}{\partial X} & 0 & \frac{\partial N_{i}}{\partial Z} \\
0 & 0 & \frac{\partial N_{i}}{\partial Z} & 0 & \frac{\partial N_{i}}{\partial X} & \frac{\partial N_{i}}{\partial Y}
\end{array}\right)^{T}
$$


Similarly, the discrete divergence operator $\nabla \cdot \gamma_{h}$ in Eq. (30) is written in matrix form as $\boldsymbol{B}_{\varepsilon}=$ $\left[\boldsymbol{B}_{1}^{T} \boldsymbol{B}_{2}^{T} \boldsymbol{B}_{3}^{T} \boldsymbol{B}_{4}^{T}\right]$.

\section{Plasticity models}

\subsection{Plasticity constitutive relation}

In small strain plasticity, the total strain $\varepsilon$ can be split into its elastic $\varepsilon_{e}$ and plastic $\varepsilon_{p}$ components

$$
\varepsilon=\varepsilon_{e}+\varepsilon_{p}
$$

so that the stress can be written as:

$$
\boldsymbol{\sigma}=\boldsymbol{C}_{0}:\left(\varepsilon-\varepsilon_{p}\right)=\boldsymbol{C}_{0}: \varepsilon_{e}
$$

where $\boldsymbol{C}_{0}$ is the elastic constitutive tensor. The elasto-plastic problem is fully defined by specifying flow rules of the type $\dot{\varepsilon}_{\boldsymbol{p}}=\dot{\varepsilon}_{p}(\boldsymbol{\sigma})$ for the plastic strain.

Alternatively, the stress can be expressed in secant form as [9]:

$$
\boldsymbol{\sigma}=\left[\boldsymbol{C}_{0}-\frac{\boldsymbol{C}_{0}: \varepsilon_{p} \otimes \boldsymbol{C}_{0}: \varepsilon_{p}}{\varepsilon: \boldsymbol{C}_{0}: \varepsilon_{p}}\right]: \varepsilon=\boldsymbol{C}(\varepsilon): \varepsilon
$$

where $\boldsymbol{C}=\boldsymbol{C}(\boldsymbol{\varepsilon})$ is the secant constitutive tensor. Either Eqs. (42) or (43) fit into the mixed strain-displacement framework described in Section 2.

\subsection{Mohr-Coulomb and Drucker-Prager plasticity models}

The Mohr-Coulomb (MC) criterion, and associated plasticity model, is used to describe the failure of frictional materials and geomaterials in general. The behavior of these materials is characterized by the dependence of the effective cohesion on pressure. The plastic strains are the result of the relative sliding and friction between particles. According to the MC criterion, plastic flow occurs when a certain combination of shear stress $\tau$ and normal stress $\sigma_{n}$ reach a critical value:

$$
|\tau|=c-\sigma_{n} \tan \phi
$$

being $c \geq 0$ the cohesion and $0 \leq \phi \leq \pi / 2$ the internal friction angle. For $\phi=0$ the MC model reduces to Tresca's model. Strain localization occurs if cohesion decreases as plastic strain grows. The MC criterion is often written as:

$$
\Phi\left(\tau, \sigma_{n}, c\left(\bar{\varepsilon}_{p}\right), \phi\right)=|\tau|-c\left(\bar{\varepsilon}_{p}\right)+\sigma_{n} \tan \phi=0
$$

where $\bar{\varepsilon}_{p}$ is the equivalent plastic strain, a scalar measure of the plastic straining. The MC criterion can also be expressed in terms of the stress invariants or, more frequently, as six planes in the HaighWestergaard space of principal stresses, where they form the characteristic hexagonal pyramid of the MC model. One of such planes is given by:

$$
\Phi_{1}\left(\sigma_{1}, \sigma_{3}, c\left(\bar{\varepsilon}_{p}\right), \phi\right)=\left(\sigma_{1}-\sigma_{3}\right)+\left(\sigma_{1}+\sigma_{3}\right) \sin \phi-2 c\left(\bar{\varepsilon}_{p}\right) \cos \phi=0
$$




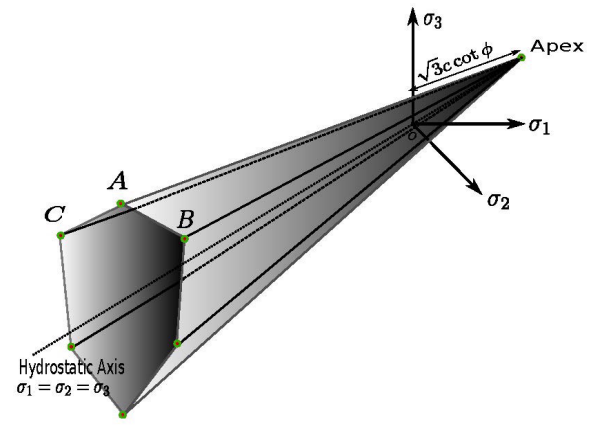

(a)

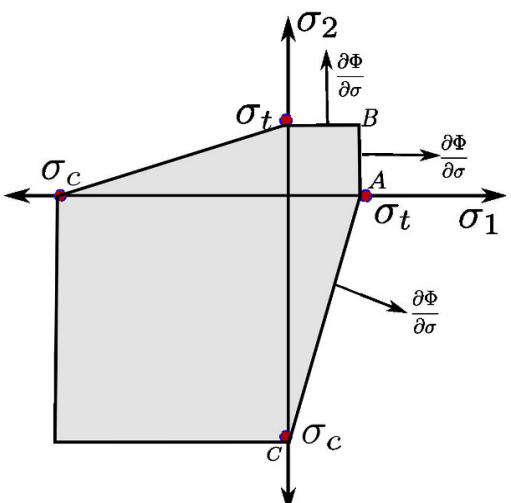

(b)

Figure 1: Mohr-Coulomb model (a) in 3D stress space (b) in plane stress.

where $\sigma_{1} \geq \sigma_{2} \geq \sigma_{3}$ are the principal stresses. Figure 1 depicts the MC pyramid in the HC space. Note that this is a multisurface criterion with the apex located on the hydrostatic axis, at $\sqrt{3} c \cot \phi$ from the origin.

The Drucker-Prager (DP) criterion was proposed as a smoothed version of the MC criterion. It is also considered as an extension of the von Mises model in which the pressure dependence is considered. In this model, plastic flow occurs when the second invariant of the deviatoric stress tensor $J_{2}=\frac{1}{2} \boldsymbol{S}: \boldsymbol{S}$ and the hydrostatic pressure reach a critical combination:

$$
\Phi\left(\boldsymbol{\sigma}, c\left(\overline{\boldsymbol{\varepsilon}}_{p}\right), \phi\right)=\sqrt{J_{2}(\boldsymbol{\sigma})}+\eta p-\varsigma c\left(\overline{\boldsymbol{\varepsilon}}_{p}\right)=0
$$

The DP criterion, Figure 2, appears in the HW space as a cone whose axis coincides with the hydrostatic axis. Note that For $\phi=0$ the DP model reduces to von Mises incompressible model. The parameters $\eta$ and $\varsigma$ are usually chosen to approximate the MC criterion in different ways. In

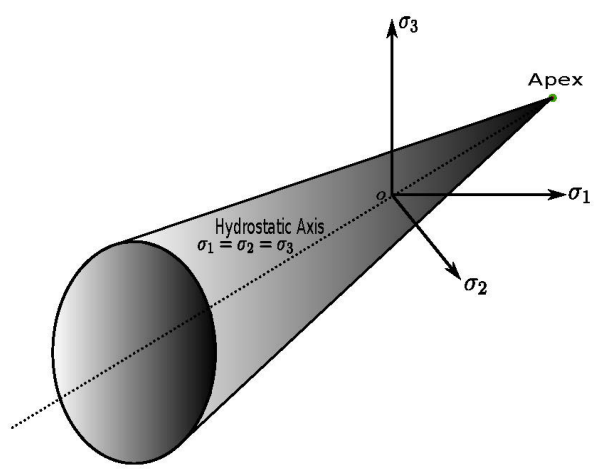

(a)

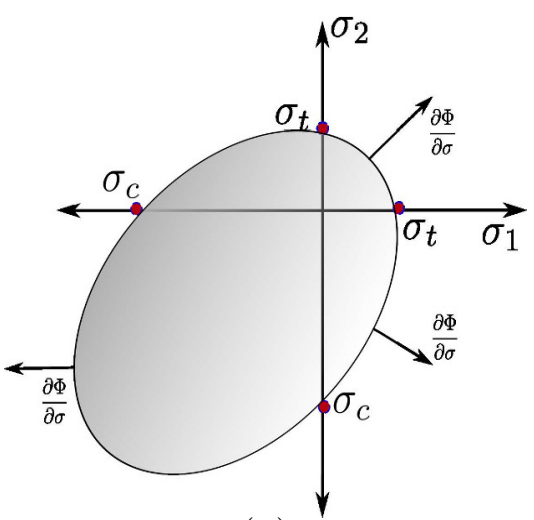

(b)

Figure 2: Drucker-Prager model (a) in 3D stress space (b) in plane stress. 
plane strain situations, to predict the same limit loads as with MC, $\eta$ and $\varsigma$ are taken as [40]:

$$
\eta=\frac{3 \tan \phi}{\sqrt{9+12 \tan ^{2} \phi}} \text { and } \varsigma=\frac{3}{\sqrt{9+12 \tan ^{2} \phi}}
$$

\subsection{Return mapping, multisurface plasticity and softening}

At each time step, the constitutive model must be integrated to update the value of the plastic strains. To this end, implicit algorithms to ensure that the stress stays in the yield surface during plastic flow are often used. These are the so-called return mapping algorithms. In multisurface associate plasticity, Koiter's rule is used to define the plastic flow and the equivalent plastic strain rate [46]:

$$
\begin{aligned}
\dot{\boldsymbol{\varepsilon}}_{p} & =\sum_{k=1}^{n_{a c t}} \dot{\lambda}_{k} \frac{\partial \Phi_{k}}{\partial \boldsymbol{\sigma}}=\sum_{k=1}^{n_{a c t}} \dot{\lambda}_{k} \boldsymbol{m}_{k} \\
\dot{\bar{\varepsilon}}_{p} & =-\sum_{k=1}^{n_{a c t}} \dot{\lambda}_{k} \frac{\partial \Phi_{k}}{\partial c}
\end{aligned}
$$

where $\dot{\lambda}_{k}$ are the plastic consistency parameters and $n_{\text {act }}$ is the number of active surfaces. The problem consists on determining $\varepsilon_{p}, \lambda_{k}$ and the active surfaces $\mathbb{J}:=\Phi_{k} \forall k \in\left\{1,2 . . n_{\text {act }}\right\}$. Also, the plastic consistency parameters must fulfill the Kuhn-Tucker conditions extended to multiple surfaces plasticity:

$$
\dot{\lambda}_{k} \geq 0, \dot{\lambda}_{k} \Phi_{k}\left(\boldsymbol{\sigma}, c\left(\bar{\varepsilon}_{p}\right)\right) \leq 0 \text { and } \dot{\lambda}_{k} \dot{\Phi}_{k}\left(\boldsymbol{\sigma}, c\left(\bar{\varepsilon}_{p}\right)\right)=0
$$

details on the procedures to integrate the MC and DP plasticity models are given in reference [40].

Furthermore, the energy dissipated during the formation of a shear band is related to the mode II fracture energy $\mathcal{G}_{f}$, defined per unit area. This must be related to the plastic work $\mathcal{W}_{p}$, defined per unit volume, dissipated during the plastic flow. Assuming that in the finite element discretization, strain localization occurs in a ban of maximum resolution, one element thick, $h$ :

$$
\mathcal{W}_{p}=\frac{\mathcal{G}_{f}}{h}
$$

The softening law for cohesion can be defined in different ways. In this work, exponential softening is considered:

$$
c=c_{0} \exp \left(\frac{-2 \mathcal{H}_{s}}{c_{0}} \bar{\varepsilon}_{p}\right) \quad 0<\bar{\varepsilon}_{p}<\infty
$$

With these softening law for cohesion, the plastic work done from the onset of plasticity $(t=0$, $c=c_{0}$ and $\left.\overline{\boldsymbol{\varepsilon}}_{p}=0\right)$ to full decohesion $\left(t=\infty, c=0\right.$ and $\left.\overline{\boldsymbol{\varepsilon}}_{p} \neq 0\right)$ is:

$$
\mathcal{W}_{p}=\int_{t=0}^{t=\infty} \dot{\mathcal{W}}_{p} d t=\int_{t=0}^{t=\infty} \sigma: \dot{\varepsilon}_{p} d t=\frac{c_{0}^{2}}{2 \mathcal{H}_{s}}
$$

Equating (52) and (54), the softening parameter $\mathcal{H}_{s}$ depends on the material properties and the size of the finite element discretization:

$$
\mathcal{H}_{s}=\frac{c_{0}^{2} h}{2 \mathcal{G}_{f}}
$$




\subsection{Orientation of the localization band}

Strain localization in geomaterials reveals as shear bands, narrow bands of intense straining bounded by surfaces where strain (weak) discontinuities occur.

Several authors $[3,6,15,34,37,43,44,45,48]$ have analytical and geometrical solutions for the orientation of discontinuity $S$ bands (see Fig. 3) in elasto-plastic materials using different strategies. All of them base their solutions on the so-called localization condition, as it is a necessary condition for the appearance of weak discontinuities and, subsequently, strain localization.

Differently, Cervera et al. [10, 14, 51] propose a different methodology to find analytical solutions for the band orientation. This procedure states the conditions for boundedness of stresses and full decohesion, which combined, are more stringent necessary conditions for the formation of the shear band. According to this, the band orientation in an associated plasticity model does not depend on the elastic constants (such as Poisson's ratio), but only on the plastic flow, determined by the yield criterion and the stress state. Table 1 shows analytical values of the localization angle of locale $\theta_{l o c}$ for the MC and DP in plane stress and plane strain situations. Cervera et al. [9] have verified these results numerically for J2 plasticity and the DP model using an implicit mixed strain/displacement formulation.

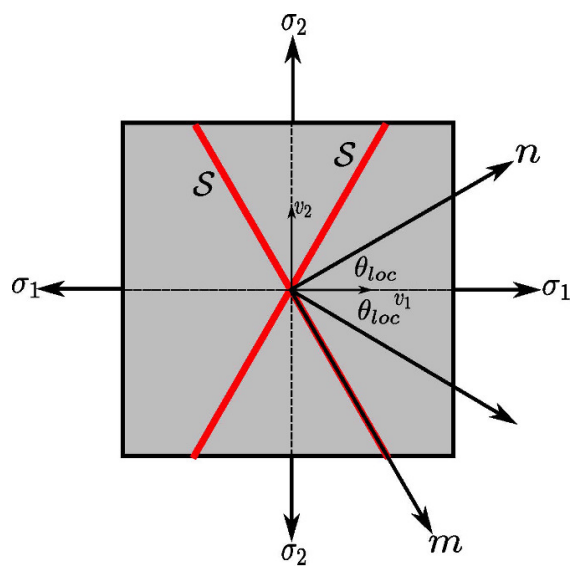

Figure 3: Definition of the localization angle

\begin{tabular}{ccc}
\hline Model & Plane stress & Plane strain \\
\hline MC & $\theta_{l o c}=\left\{\begin{array}{cc}0^{o} & \text { if flow normal to plane } A-B \\
\pm\left(45^{o}-\frac{\phi}{2}\right) & \text { if flow normal to plane } A-C\end{array}\right.$ & $\theta_{l o c}= \pm\left(45^{o}-\frac{\phi}{2}\right)$ \\
DP & $\tan ^{2} \theta_{l o c}=\frac{\sigma_{1}-2 \sigma_{2}-2 J_{2}^{1 / 2} \eta}{2 \sigma_{1}-\sigma_{2}+2 J_{2}^{1 / 2} \eta}$ & $\tan ^{2} \theta_{l o c}=\frac{\sigma_{1}-\sigma_{2}-2 J_{2}^{1 / 2} \eta}{\sigma_{1}-\sigma_{2}+2 J_{2}^{1 / 2} \eta}$ \\
\hline
\end{tabular}

Table 1: Analytical localization angles for MC and DP in plane stress and plane strain 


\section{Numerical Examples}

In this Section, the efficiency and robustness of the proposed explicit stabilized mixed straindisplacement formulation (MEX-FEM) are demonstrated by solving several benchmark tests. The different problems involve compressible and quasi-incompressible elasticity in dynamic and quasistatic conditions and perfect and softening plasticity with strain localization. They are also solved in $2 \mathrm{D}$ and $3 \mathrm{D}$.

Simulations are performed with an enhanced version of the finite element program KRATOS $[24,25]$, developed at the International Center for Numerical Methods in Engineering (CIMNE). Pre and post-processing is done with GiD, also developed at CIMNE [28]. The reference solutions for the 2D and 3D Cook's membrane have been obtained with fine structured meshes of 4- and 8-noded quadrilateral elements with constant pressure and implicit HHT time integration.

\subsection{Cook's membrane. Compressible and quasi-incompressible elasticity}

The proposed MEX-FEM formulation is first tested numerically on Cook's membrane, with the geometry shown in Figure 4. The problem is analyzed in 2D, under plane strain assumptions. Four finite element meshes are used, with progressive level of refinement, see Figure 5. Both compressible and quasi-incompressible cases are considered with elastic properties: Young's modulus $E=200 \mathrm{MPa}$ and Poisson's ratio $\nu=0.30$ and $\nu=0.499$. Material density is $\rho=10 \mathrm{~kg} / \mathrm{m}^{3}$.

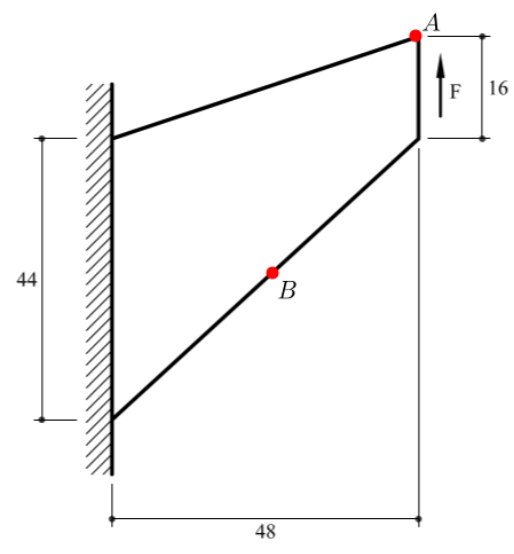

Figure 4: Cook's membrane. Geometry (units in mm)

Firstly, the transient dynamic analysis of the problem is addressed. In this case, the load $F$ at the free end of the short cantilever is applied instantly at $t=0$, and it remains constant in time. For the integration in time, the time step is selected so that conditional stability is warranted [35]. The OSS method is used for the stabilization of the mixed problem, with algorithmic constants $c_{u}=1.0, c_{\varepsilon}=1.0$ and $L_{0}=50 \mathrm{~mm}$. In $2 \mathrm{D}$, the size of the element is computed as $h=(4 / \pi \cdot A)^{\frac{1}{2}}$, $A$ being the area of the element. The displacement subscale $\widetilde{\boldsymbol{u}}$ is computed using Eq. (40). Mass and stiffness proportional damping with $\alpha \Delta t=0.1$ and $\beta=10^{-7}$ is used to filter out spurious high frequencies; this damping is not emerge in the low frequency response. 


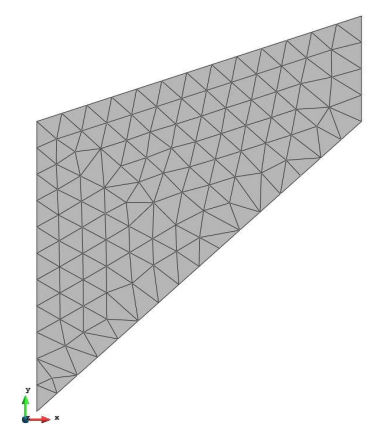

Mesh A. Nn: 123

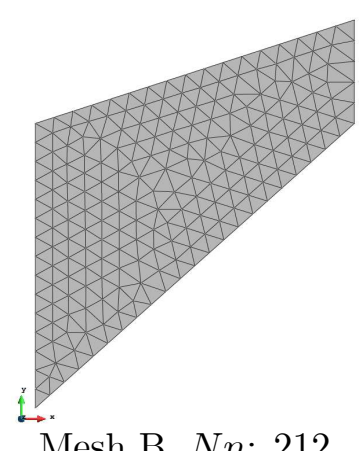

Mesh B. Nn: 212
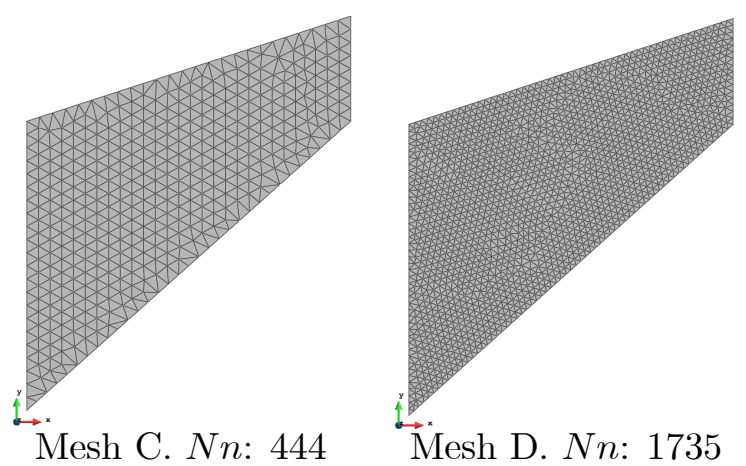

Figure 5: Cook's membrane. Meshes used for 2D analysis. $N n$ : number of nodes

Figure 6 compares results obtained for the evolution of the vertical displacement at point $\mathrm{A}$ and the pressure at point $\mathrm{B}$, for the compressible case, Poisson's ratio $\nu=0.3$ using both the irreducible and the MEX-FEM formulation. As the pressure field is discontinuous for the $P 1$ linear irreducible element, a continuous field is obtained for comparison purposes, using the $\mathcal{L}_{2}$ projection, so that $p_{h}=\mathcal{P}_{h}(p)$. Satisfactory results are obtained for the displacements in all cases (top row); the rate of convergence for the pressure is noticeably lower in the irreducible case and very similar to that of the displacements for the mixed form.

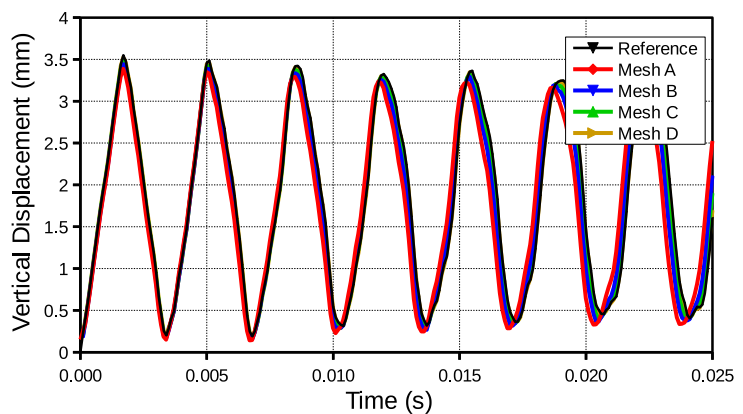

Vertical displacement at point A. IRR

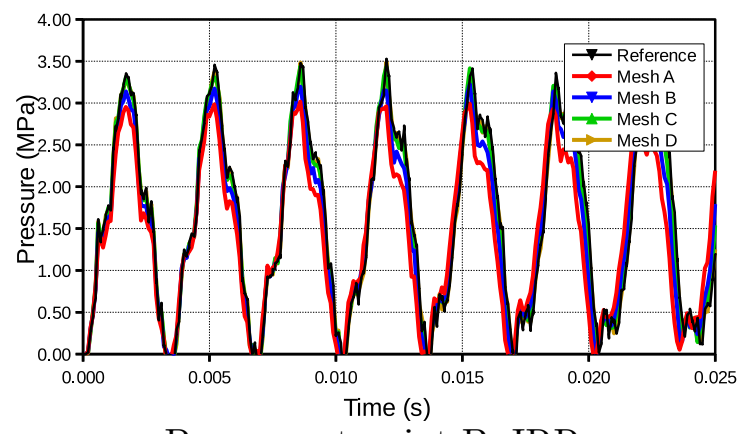

Pressure at point B. IRR

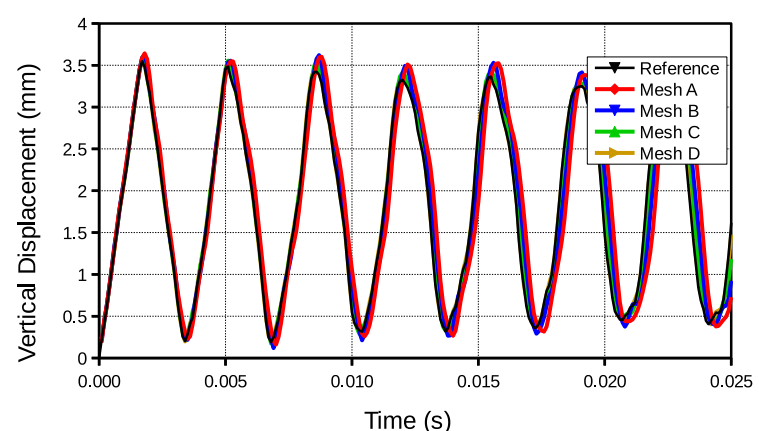

Vertical displacement at point A. MIXED

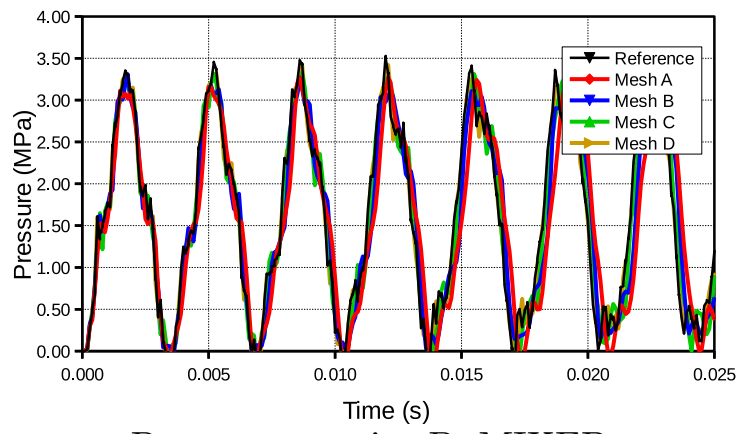

Pressure at point B. MIXED

Figure 6: Cook's membrane in 2D. Results for irreducible and mixed formulations. $\nu=0.30$ 


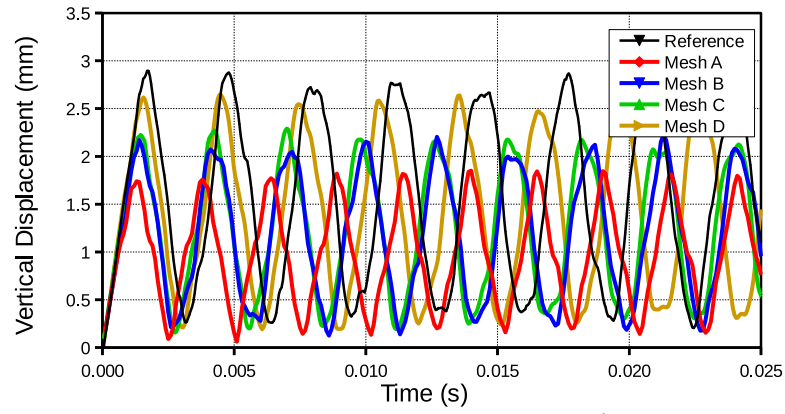

Vertical displacement at point A. IRR

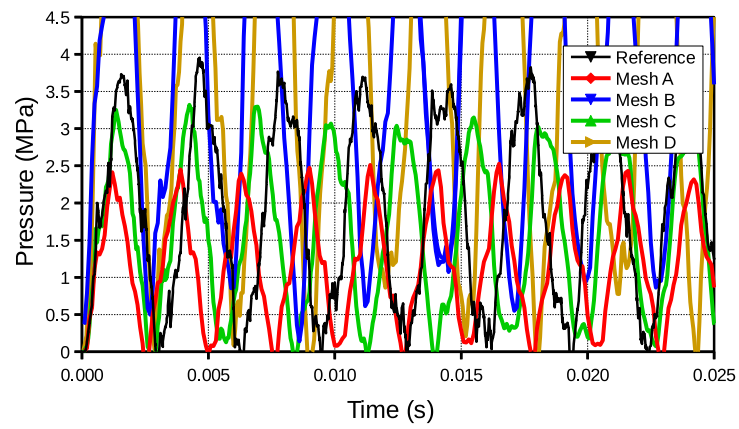

Pressure at point B. IRR

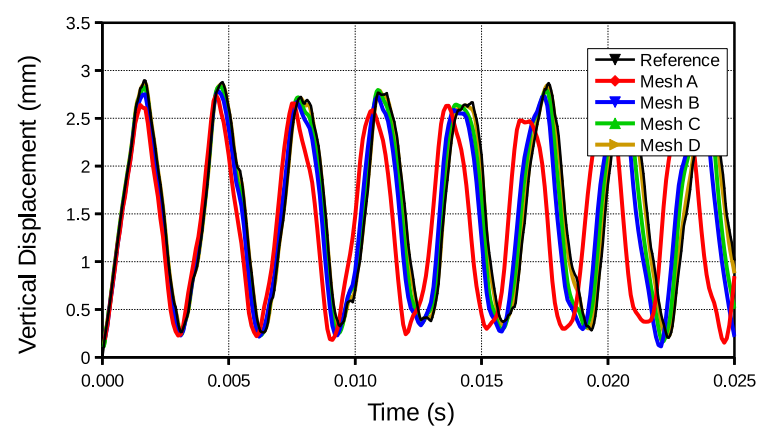

Vertical displacement at point A. MIXED

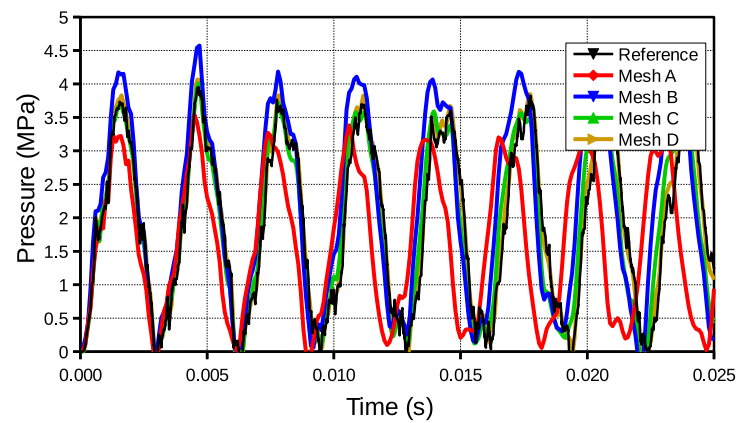

Pressure at point B. MIXED

Figure 7: Cook's membrane in 2D. Results for irreducible and mixed formulations. $\nu=0.499$

Figure 7 shows corresponding results for the quasi-incompressible case, Poisson's ratio $\nu=0.499$. Here, the advantage of the proposed mixed formulation is evident. The irreducible formulation, on the one hand, grossly underestimates the vertical displacements, even for rather fine meshes; the phase error is also unacceptable. On the other hand, the pressure field is highly unstable. Results obtained with the present mixed formulation are remarkably better, regarding amplitude and phase, both in the displacement and pressure fields.

Next, the stationary problem is solved using a pseudo-transient analysis with over-damping to achieve a steady-state solution. The relative rate of convergence of the different formulations is shown in Figures 8 and 9, for the compressible and quasi-incompressible cases, respectively. Results are also shown for the MEX-FEM formulation without displacement subscale, $\widetilde{\boldsymbol{u}}=\mathbf{0}$ as originally presented in reference [35].

As expected, for the $\nu=0.3$ case, all the formulations converge satisfactorily to the correct values $(1.843 \mathrm{~mm}$ for the displacement and $1.632 \mathrm{MPa}$ for the pressure)at the expected rate, that of the mixed formulations higher for the pressure field. However, for the $\nu=0.499$ case, only the mixed formulations converge satisfactorily to the correct values $(1.554 \mathrm{~mm}$ for the displacement and $1.872 \mathrm{MPa}$ for the pressure). Furthermore, the irreducible formulation and the mixed one without the displacement subscale produce erratic results for the pressure.

Finally, Figure 10 shows pressure field contours obtained on mesh $D$ for the case $\nu=0.499$. Note that only the proposed MEX-FEM formulation is able to eliminate the pressure instability, thus indicating the betterment of the displacement subscale in quasi-incompressible situations. 


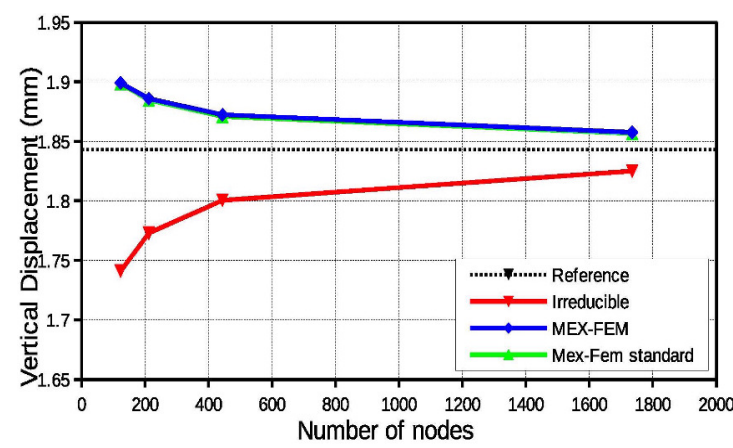

(a) Vertical displacement at point A

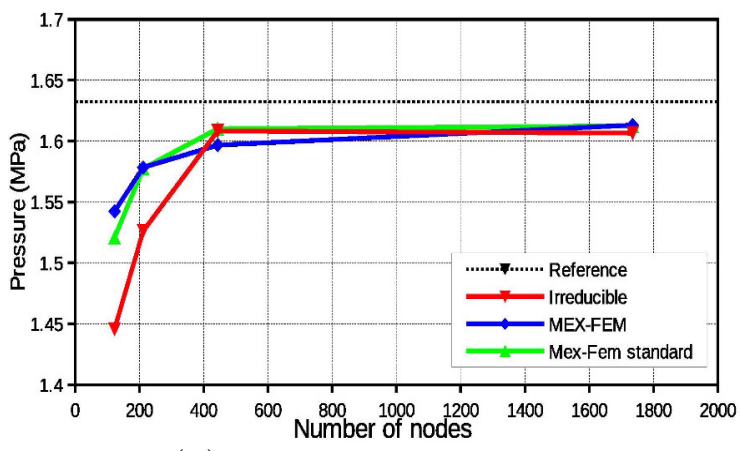

(b) Pressure at point B

Figure 8: Cook's membrane in 2D. Convergence curves for $\nu$ : 0.30 .

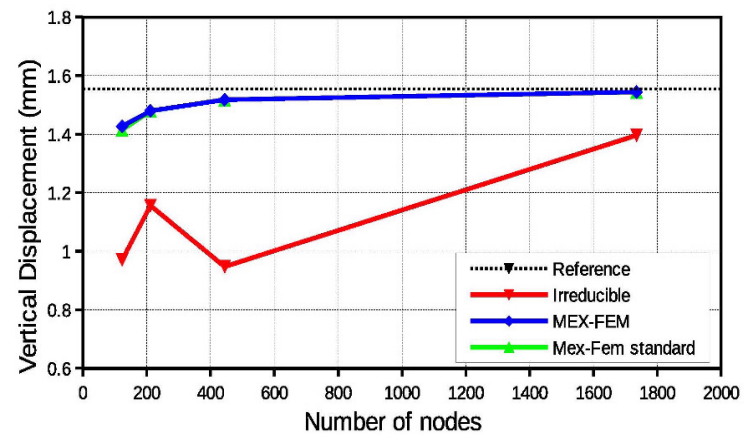

(a) Vertical displacement at point A

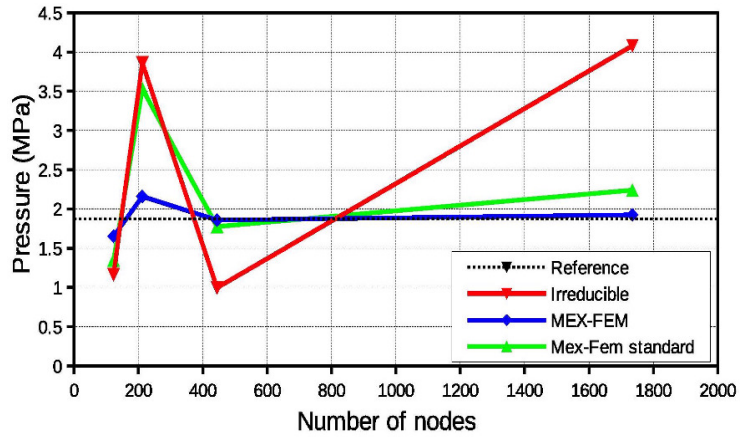

(b) Pressure at point B

Figure 9: Cook's membrane in 2D. Convergence curves for $\nu=0.499$.

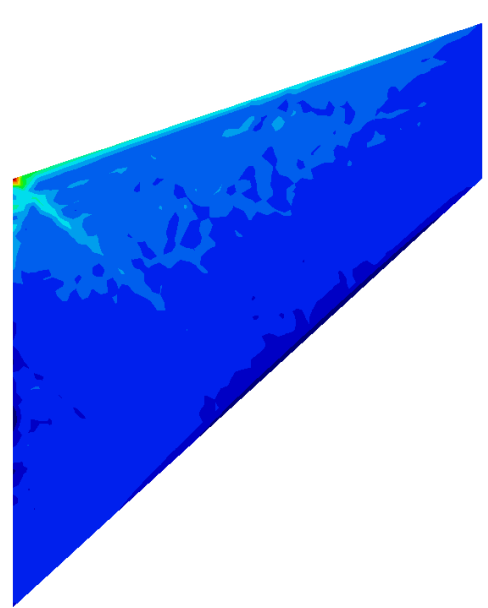

(a) Irreducible

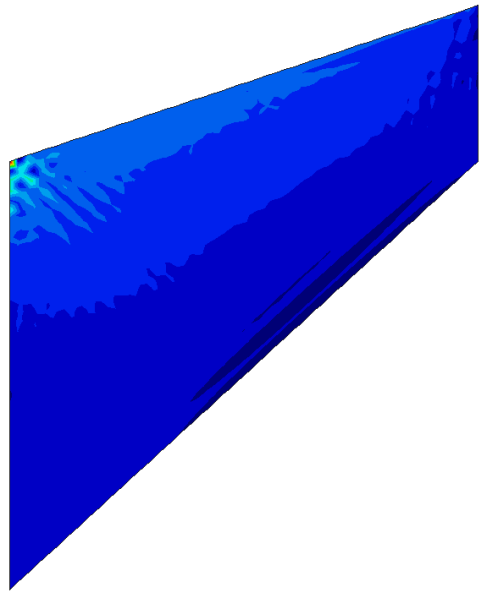

(b) Mixed, no displ. subs.

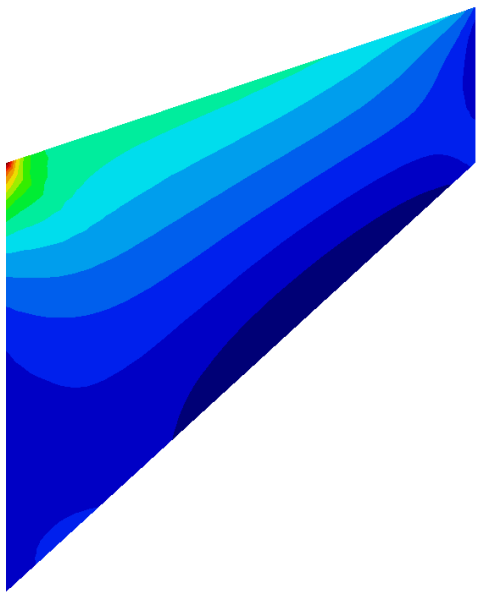

(c) Mixed with displ. subs.

Figure 10: Cook's membrane in 2D. Pressure contours in Mesh D. $\nu=0.499$ 

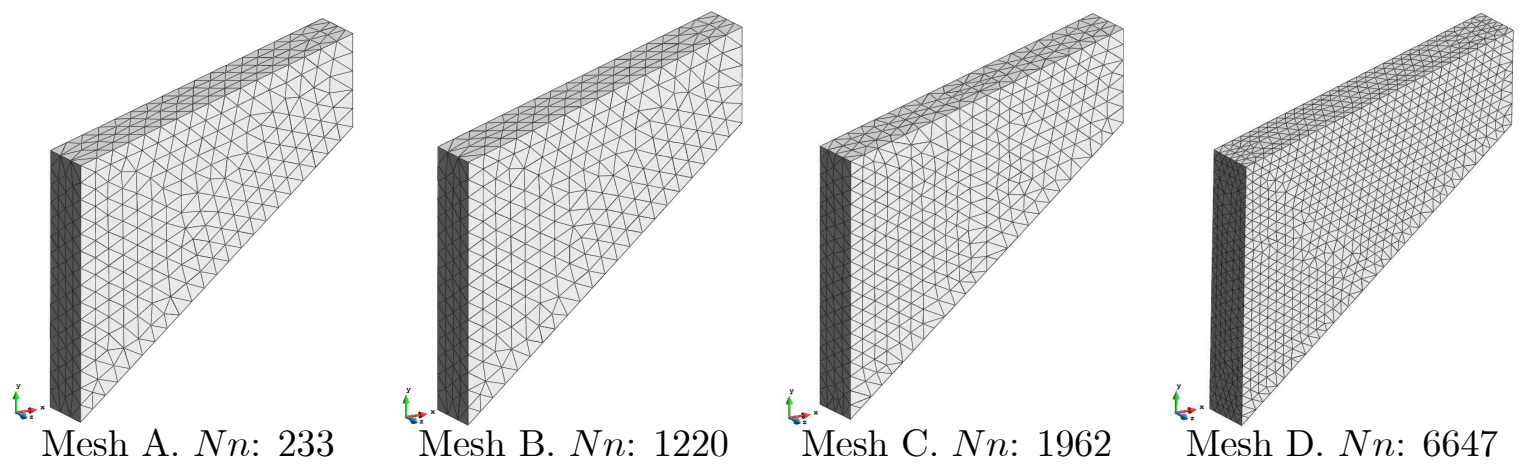

Figure 11: Cook's membrane. Meshes used for 3D analysis. $N n$ : number of nodes

\subsection{Cook's membrane. Quasi-incompressible elasticity in 3D}

In this Section, the Cook membrane problem in Figure 5 is analyzed in 3D (thickness $10 \mathrm{~mm}$ ) for quasi-incompressible elastic properties, Poisson's ratio $\nu=0.499$. Again, four finite element meshes are used, with progressive level of refinement, see Figure 11. In the tridimensional case, the size of the element is taken as $h=(6 / \pi \cdot V)^{\frac{1}{3}}$, being $V$ the element volume. Time integration, numerical damping and stabilization parameters are selected as for the $2 \mathrm{D}$ case.
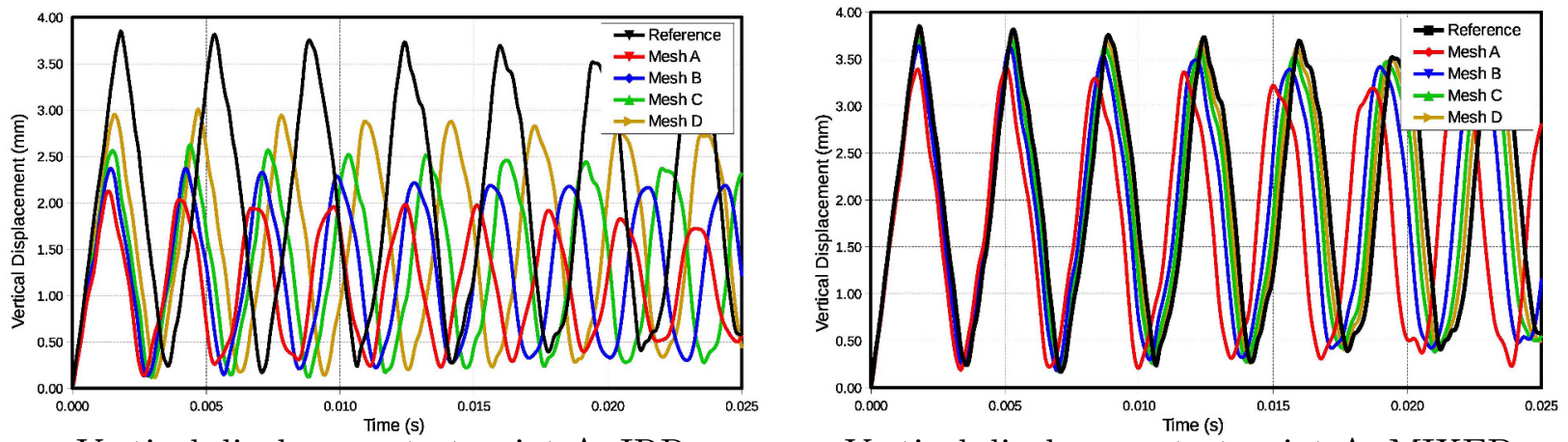

Vertical displacement at point A. IRR
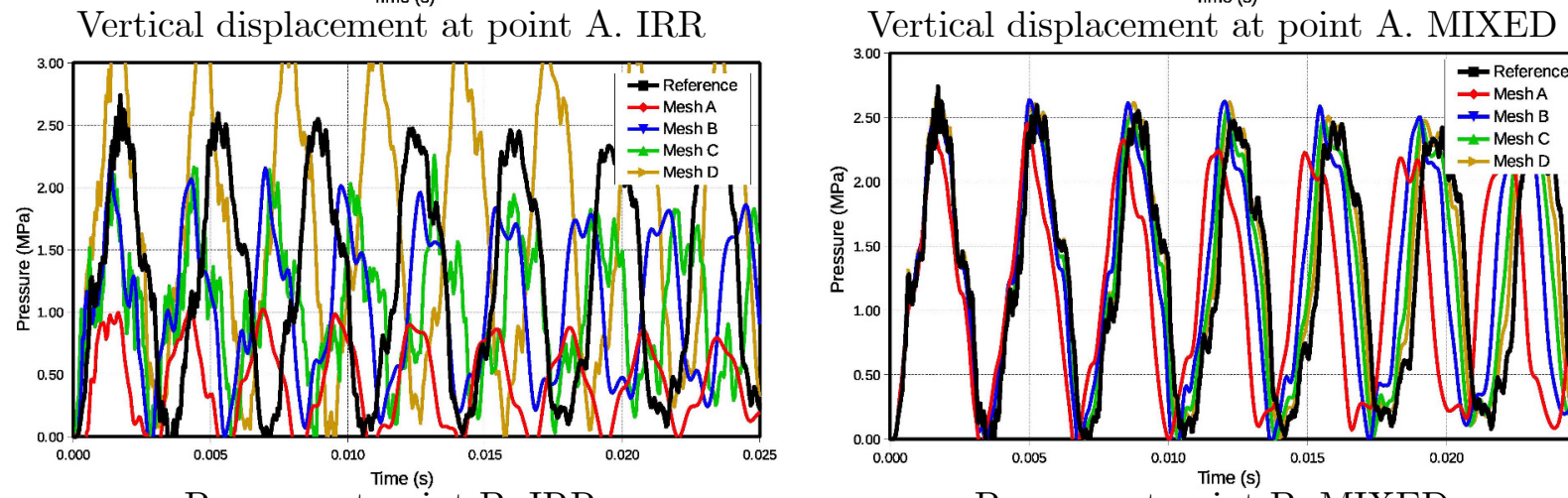

Pressure at point B. IRR

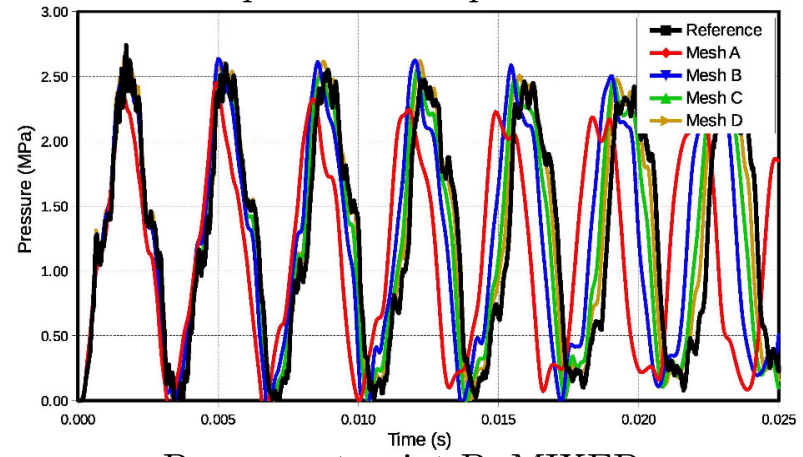

Pressure at point B. MIXED

Figure 12: Cook's membrane in 3D. Results for irreducible and mixed formulations. $\nu=0.499$ 


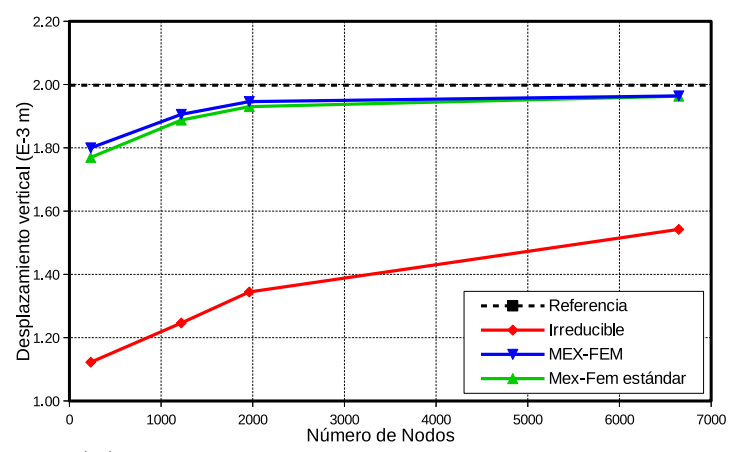

(a) Vertical displacement at point $\mathrm{A}$

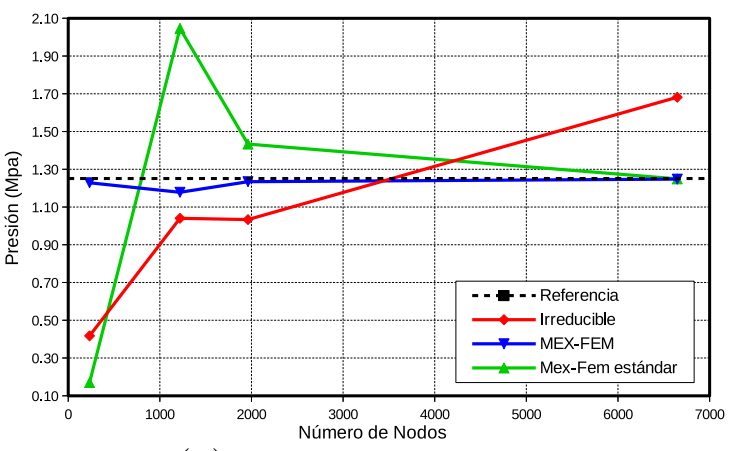

(b) Pressure at point b

Figure 13: Cook's membrane in 3D. Convergence curves for $\nu=0.499$.

Results for the tridimensional dynamic analysis are shown in 12 . Again, the irreducible $P 1$ element shows an extremely poor performance due to volumetric locking. The computed displacements are underestimated, even in relatively fine meshes. Phase error is also severe. Pressure is also unstable. Contrarily, the MEX-FEM formulation shows satisfactory results both in terms of displacements and pressure.

Next, the problem is solved using a pseudo-transient analysis with over-damping to achieve a steady-state solution. The relative rate of convergence of the different formulations for the stationary problem is shown in Figure 13. Again, the MEX-FEM with displacement subscale is the only one capable of converging to the correct values $(1.998 \mathrm{~mm}$ for the vertical displacement and 1.251 MPa for the pressure).

Finally, Figure 14 shows pressure contours for mesh $D$ using three different formulations. Again, only the MEX-FEM formulation with displacement subscale is able to eliminate the pressure instability.

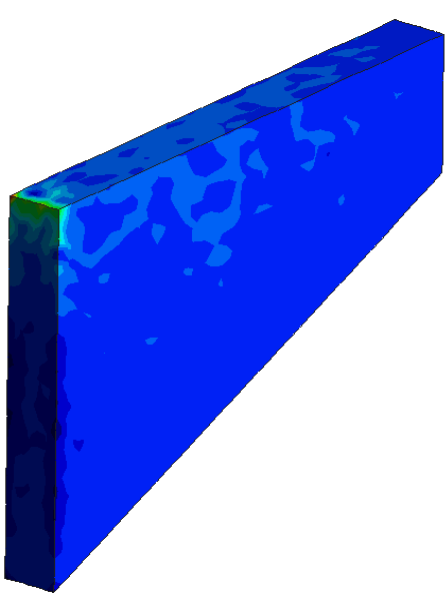

(a) Irreducible

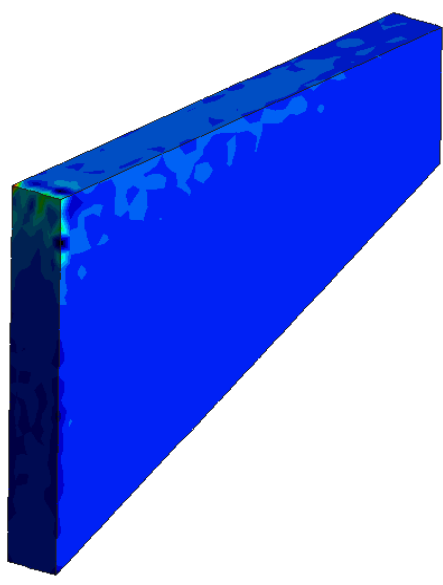

(b) Mixed, no displ. subs.

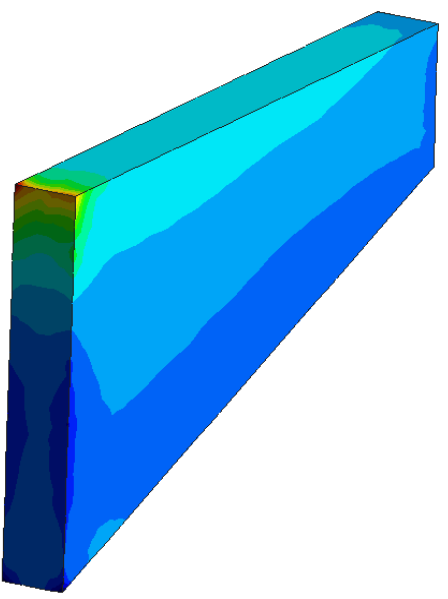

(c) Mixed with displ. subs.

Figure 14: Cook's membrane in 3D. Pressure contours in Mesh D. $\nu$ : 0.499 


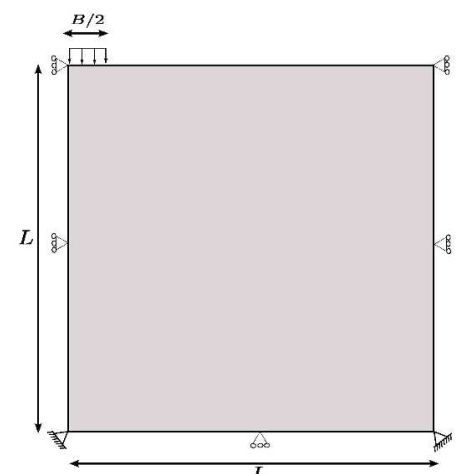

(a)

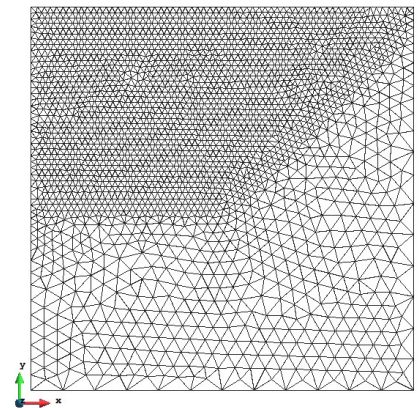

(b)

Figure 15: Prandtl's punch test. (a) Geometry (b) FE mesh

\subsection{Prandtl's punch test. Incompressible perfect plasticity}

This 2D example in plane strain is often used to assess the computation of failure mechanisms and failure loads in plasticity models. Figure 15 shows the geometry and the mesh used in the present analysis. Because of symmetry, only half of the domain (the right half) is considered. Dimensions are: $L=5 \mathrm{~m}$ and $B=1 \mathrm{~m}$. The OSS method is used for the stabilization of the mixed problem, with algorithmic constants $c_{u}=1.0, c_{\varepsilon}=1.0$ and $L_{0}=5 \mathrm{~m}$. The domain is discretized with a non-structured mesh of 2438 nodes and 4731 linear elements, both for the irreducible $(P 1)$, and the MEX-FEM $(P 1 P 1)$ formulations. The problem is also analyzed with quadratic irreducible elements $(P 2)$. In all cases a constant velocity of $10^{-3} \mathrm{~m} / \mathrm{s}$ is imposed in the indicated area. The quasi-static problem is solved using mass proportional damping, with $\alpha \Delta t=0.1$.

Material properties are: density $\rho=10^{4} \mathrm{Kg} / \mathrm{m}^{3}$, Young's modulus $E=10^{7} \mathrm{KPa}$, Poisson's ratio $\nu=0.48$, initial cohesion $c_{0}=490 \mathrm{KPa}$ and friction angle $\phi=20^{\circ}$. Both the perfectly plastic models of Mohr-Coulomb and Drucker-Prager are used; for the latter, parameters $\eta$ and $\varsigma$ chosen as indicated in Eq. (48). With these values, the analytical solution for the quasi-static problem is $P_{\text {lim }} / c_{0}=14.8$, where $P_{\text {lim }}$ is the total applied force to impose the velocity.

Figure 16 shows contours for the equivalent plastic strain obtained with the MC model; the DP model produces very similar results. The collapse mechanism obtained using the proposed MEXFEM formulation is very well defined. It coincides with the analytical solution and it is independent from the orientation of the mesh. Contrariwise, the solution obtained with the linear irreducible elements is strongly dependent on the mesh bias and it goes associated to severe stress-locking and pressure oscillations. Using quadratic irreducible elements slightly improves the solution, but the pressure oscillations are not fully eliminated.

Figure 17 shows the force-displacement curves for the different FE formulations and the two plasticity models considered. Note that MEX-FEM captures satisfactorily the peak load, with values of $P_{\text {lim }} / c_{0}=15.16$ for the $\mathrm{MC}$ model and $P_{\text {lim }} / c_{0}=15.22$ for the DP model, both in close agreement with the analytical value for the perfectly quasi-static problem. For the linear $F I-P 1$ element, the volumetric locking is evident, and the peak load can not be identified. For the quadratic $F I-P 2$ element, the limit load is $P_{l i m} / c_{0}=15.56$, somewhat overestimated. 

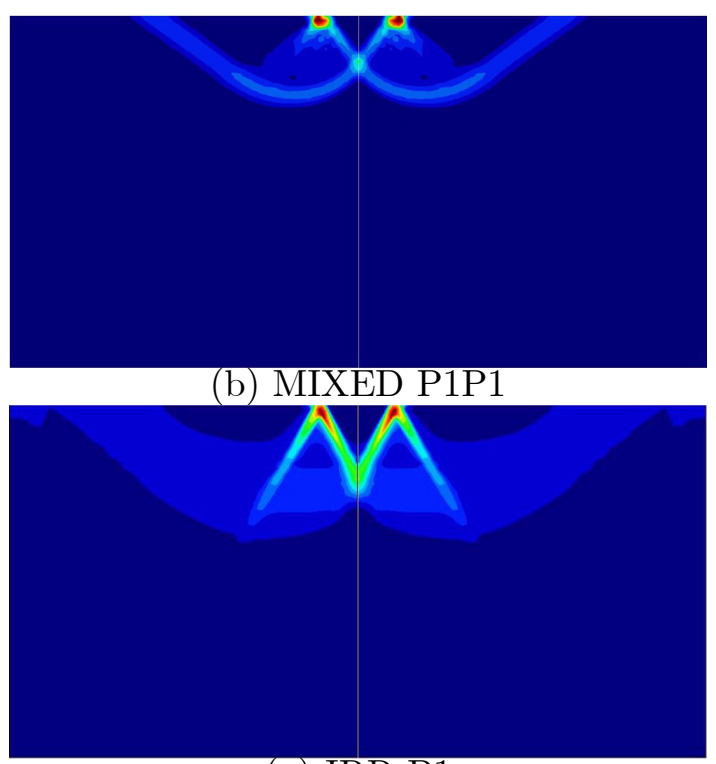

(a) IRR P1

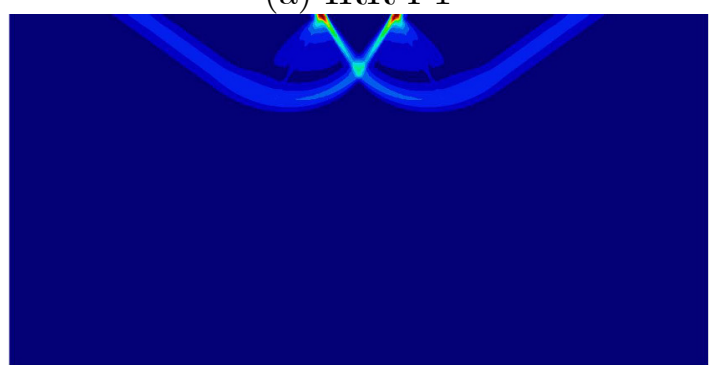

(c) IRR P2

Figure 16: Prandtl's punch test. Equivalent plastic strain for different formulations

\subsection{Perforated strip under tension. Strain localization in plane strain and stress}

This 2D example consists in a simply perforated strip subjected to uniform tension, by applying uniform normal tractions at the free ends of the strip. The objectives of the benchmark are threefold: 1) to determine numerically the localization angle $\theta_{l o c}$ for varying friction angles $\phi$, for the MC and DP models 2) to investigate the effect of the Poisson's ratio in the localization angle and 3 ) to compare the results obtained numerically with the analytical solutions proposed in Table 1.

Dimensions are $l=40 \mathrm{~m}, b=20 \mathrm{~m}$ and $d=2 \mathrm{~m}$. Thickness is $1 \mathrm{~m}$. Material properties are: Young's modulus $E=10 \mathrm{MPa}$, Poisson's ratio $\nu=\{0,0.15,0.30\}$, initial cohesion $c_{0}=10^{4} \mathrm{~Pa}$, friction angle $\phi=\left\{0^{\circ}, 15^{\circ}, 30^{\circ}, 45^{\circ}, 60^{\circ}\right\}$.

Given the symmetry of the problem, only one quarter of the domain (top-right) is considered. This is discretized with 3758 nodes and 7274 elements. Figure 18 shows the geometry of the problem and the computational mesh. The OSS method is used for the stabilization of the mixed problem, with algorithmic constants $c_{u}=1.0, c_{\varepsilon}=1.0$ and $L_{0}=10 \mathrm{~m}$. The problem is analyzed both in plane strain and plane stress conditions.

The plane strain case is considered first. The results from the analyses are presented in Table 


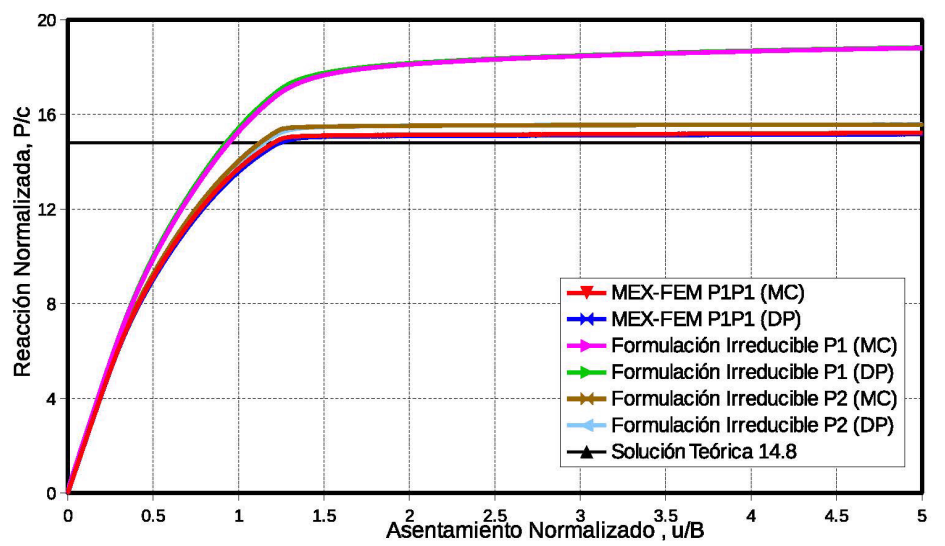

Figure 17: Prandtl's punch test. Load-displacement curves for different FE formulations

\begin{tabular}{ccccc}
\hline Friction Angle & $\begin{array}{c}\text { Analytical } \\
\theta_{l o c} D P\end{array}$ & $\begin{array}{c}\text { Numerical } \\
\theta_{l o c} D P\end{array}$ & $\begin{array}{c}\text { Analytical } \\
\theta_{l o c} M C\end{array}$ & $\begin{array}{c}\text { Numerical } \\
\theta_{l o c} M C\end{array}$ \\
\hline $0^{0}$ & $45^{\circ}$ & $44.12^{\circ}$ & $45^{\circ}$ & $44.54^{\circ}$ \\
$15^{0}$ & $36.40^{\circ}$ & $36.35^{\circ}$ & $37.5^{\circ}$ & $37.38^{\circ}$ \\
$30^{0}$ & $28.15^{\circ}$ & $29.65^{\circ}$ & $30^{\circ}$ & $30.25^{\circ}$ \\
$45^{0}$ & $20.44^{\circ}$ & $23.29^{\circ}$ & $22.5^{\circ}$ & $23.29^{\circ}$ \\
$60^{\circ}$ & $13.28^{\circ}$ & $15.52^{\circ}$ & $15^{\circ}$ & $15.52^{\circ}$ \\
\hline
\end{tabular}

Table 2: Analytical and numerical localization angles for uniaxial tension in plane strain

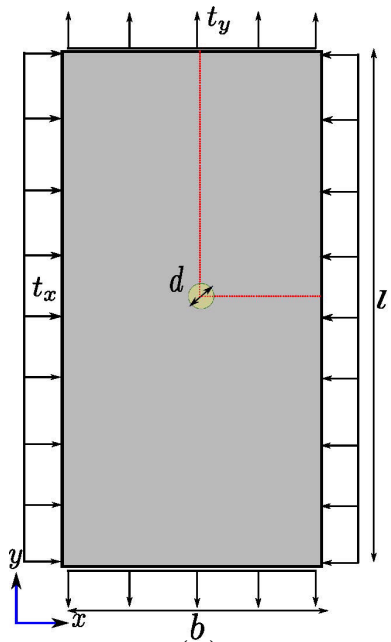

(a)

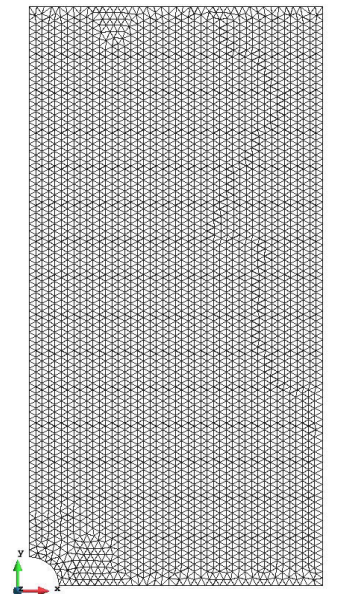

(b)

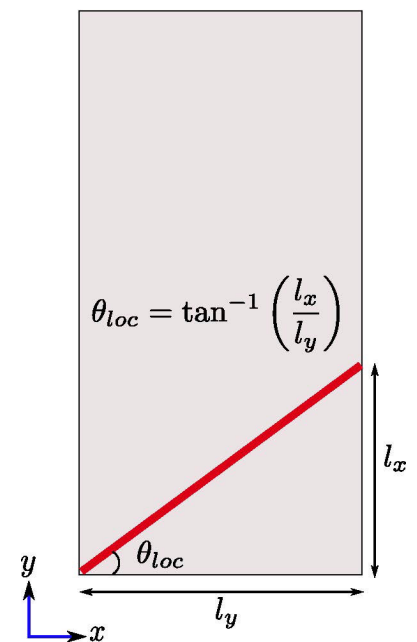

(c)

Figure 18: Perforated strip. (a) Geometry, (b) FE mesh and (c) localization angle 


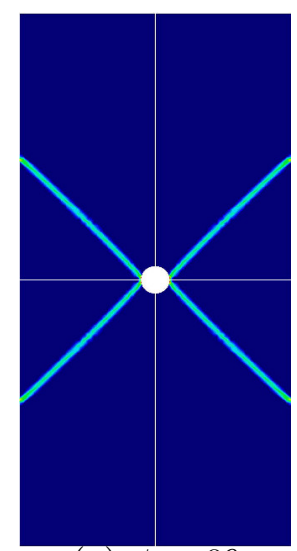

(a) $\phi=0^{\circ}$

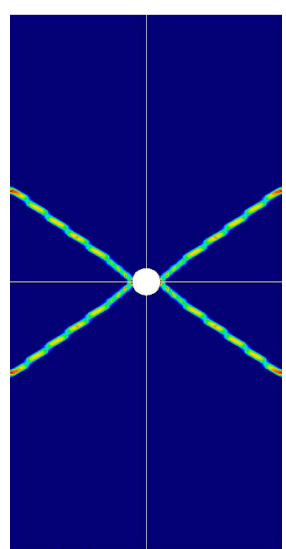

(b) $\phi=15^{\circ}$

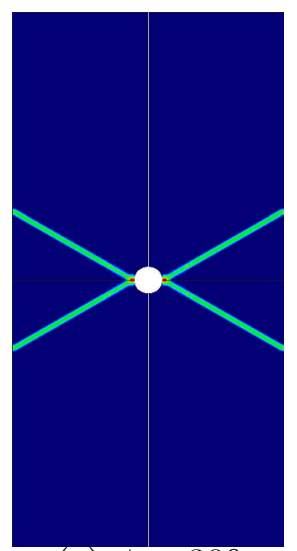

(c) $\phi=30^{\circ}$

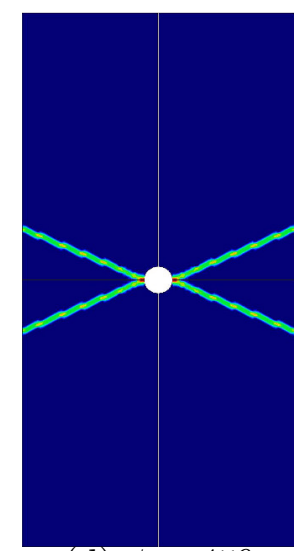

(d) $\phi=45^{\circ}$

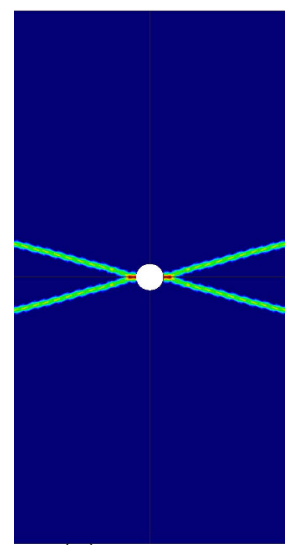

(e) $\phi=60^{\circ}$

Figure 19: Perforated strip. Plane strain. MC model. Equivalent plastic strain for different friction angles

2. The ability of the MEX-FEM formulation to capture the correct direction for the localization band, both for the MC and the DP models is remarkably demonstrated, in close agreement with the analytical predictions.

Figure 19 shows the calculated localization bands for different friction angles $\phi$, using the MC model, and represented as equivalent plastic strain contours. The collapse mechanisms are completely independent of the mesh used, the same in all cases. For the DP model, similar results are obtained, see Table 2.

\begin{tabular}{cccccc}
\hline Friction Angle & Poisson's ratio & Analytical & Numerical & Analytical & Numerical \\
$\phi$ & $\nu$ & $\theta_{l o c} D P$ & $\theta_{l o c} D P$ & $\theta_{l o c} M C$ & $\theta_{l o c} M C$ \\
\hline $0^{0}$ & 0.0 & $45^{\circ}$ & $44.94^{\circ}$ & $45^{\circ}$ & $44.94^{\circ}$ \\
$0^{0}$ & 0.15 & $45^{o}$ & $44.12^{\circ}$ & $45^{\circ}$ & $44.94^{\circ}$ \\
$30^{0}$ & 0.0 & $28.15^{\circ}$ & $29.65^{O}$ & $30^{\circ}$ & $29.65^{\circ}$ \\
$30^{0}$ & 0.15 & $28.15^{\circ}$ & $29.65^{\circ}$ & $30^{\circ}$ & $29.65^{\circ}$ \\
\hline
\end{tabular}

Table 3: Analytical and numerical localization angles in plane strain for different Poisson's ratios

Table 3 compares the numerical results obtained in plane strain for two different values of Poisson's ratio. It can be seen that the localization angle depends only on the plastic flow, as predicted analytically.

The plane stress case is now considered. Table 4 compares the localization angles obtained with the DP model in plane stress. Again, the agreement with the analytical predictions is remarkable, independently of mesh bias. Figure 20 shows some of the obtained fields of equivalent plastic strain. Note that for the DP model, the localization angle is substantially different in plane stress and plane strain.

The MC model in plane stress is now considered. For plane stress, the pure tension case in the MC model corresponds to one of the lines where two of the planes defining the yield criterion intersect, see Figure 1. Therefore, two alternative solutions exist, with the plastic flow being normal 


\begin{tabular}{ccc}
\hline Friction Angle & $\begin{array}{c}\text { Analytical } \\
\theta_{l o c} D P\end{array}$ & $\begin{array}{c}\text { Numerical } \\
\theta_{\text {loc }} D P\end{array}$ \\
\hline $0^{0}$ & $35.26^{\circ}$ & $35.25^{\circ}$ \\
$15^{0}$ & $28.98^{\circ}$ & $30.06^{\circ}$ \\
$30^{0}$ & $22.66^{\circ}$ & $24.07^{\circ}$ \\
$45^{0}$ & $16.57^{\circ}$ & $18.88^{\circ}$ \\
\hline
\end{tabular}

Table 4: Analytical and numerical localization angles for tension in plane stress for DP model

\begin{tabular}{cccc}
\hline Friction Angle & Stress & Analytical & Numerical \\
$\phi$ & state & $\theta_{l o c} M C$ & $\theta_{l o c} M C$ \\
\hline $0^{0}$ & $\boldsymbol{t}_{y}>0$ and $\boldsymbol{t}_{x}>0$ & $0^{\circ}$ & $0.00^{\circ}$ \\
$0^{0}$ & $\boldsymbol{t}_{y}>0$ and $\boldsymbol{t}_{x}<0$ & $45^{\circ}$ & $44.95^{\circ}$ \\
$30^{0}$ & $\boldsymbol{t}_{y}>0$ and $\boldsymbol{t}_{x}>0$ & $0^{\circ}$ & $0.00^{\circ}$ \\
$30^{0}$ & $\boldsymbol{t}_{y}>0$ and $\boldsymbol{t}_{x}<0$ & $30^{\circ}$ & $30.84^{\circ}$ \\
\hline
\end{tabular}

Table 5: Analytical and numerical localization angles for tension in plane stress for MC model

to plane $A-C$ or $A-B$, respectively. In order to obtain one or the other solution, the applied state of stress is perturbed by lateral normal stress of $1 \mathrm{KPa}$, applied either in compression $\boldsymbol{t}_{x}<0$ or in tension $\boldsymbol{t}_{x}>0$. For $\boldsymbol{t}_{x}>0$, strain localizes in a horizontal band, independent from the friction angle, as it would occur for a Rankine model; however, for $\boldsymbol{t}_{x}<0$, localization depends on the friction angle. Table 5 compares the numerical results obtained with MEX-FEM with the analytical predictions. Figure 21 compares the localization bands obtained for two different friction angles in the two perturbed situations.

Figures 22 show the force-displacement curves obtained for the MC model in plane strain and

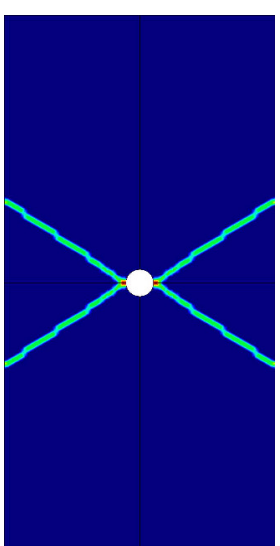

(a) $\phi=0^{\circ}$

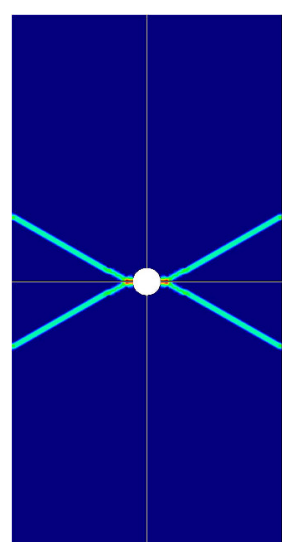

(b) $\phi=15^{\circ}$

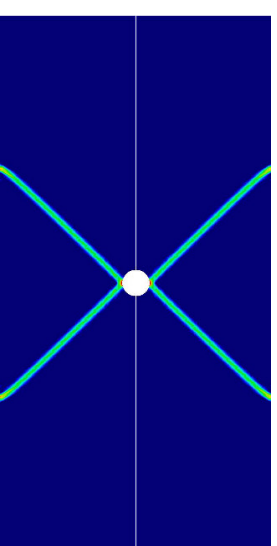

(c) $\phi=0^{\circ}$

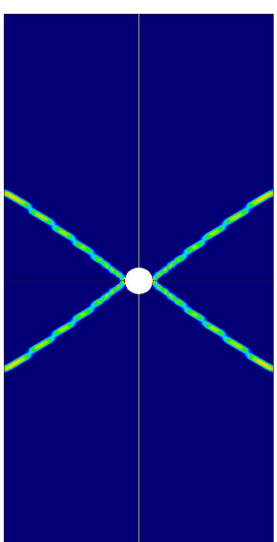

(d) $\phi=15^{\circ}$

Figure 20: Perforated strip. DC model. Equivalent plastic strain for different friction angles. (a) - (b) Plane stress, (c) - (d) Plane strain 


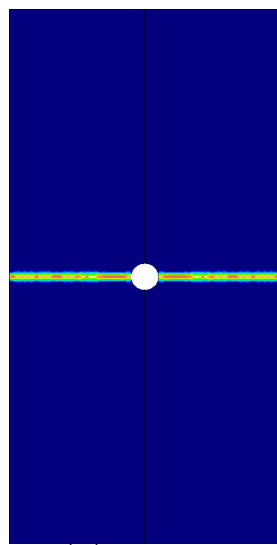

(a) $\phi=0^{\circ}$

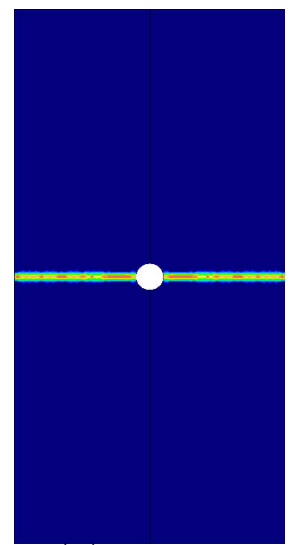

(b) $\phi=30^{\circ}$

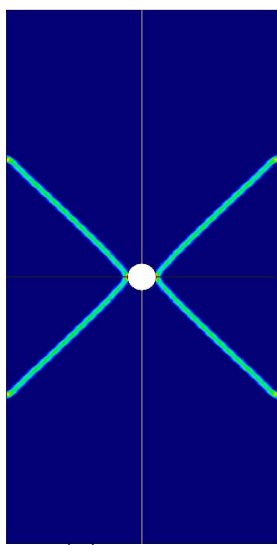

(c) $\phi=0^{\circ}$

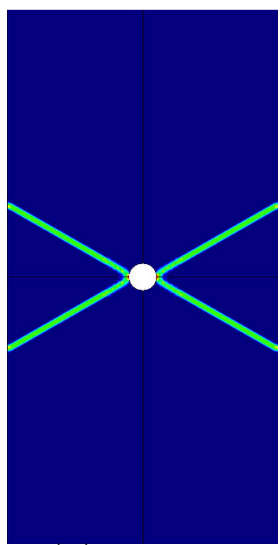

(d) $\phi=30^{\circ}$

Figure 21: Perforated strip. Plane stress. MC model. Equivalent plastic strain for different friction angles. (a) - (b) 1st stress quadrant, (c) - (d) 4th stress quadrant

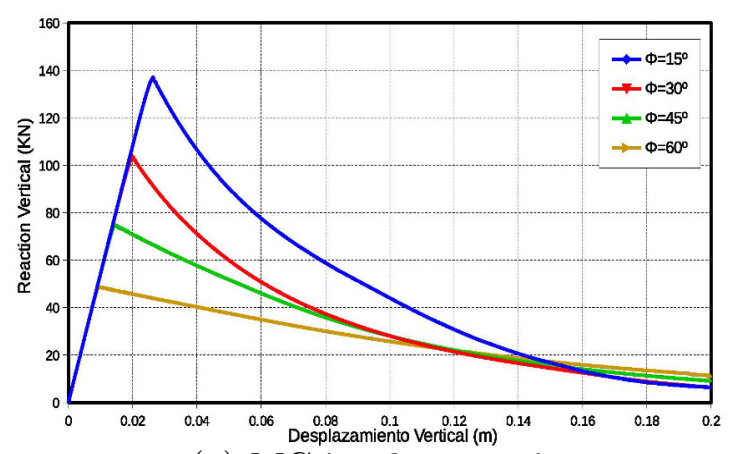

(a) MC in plane strain

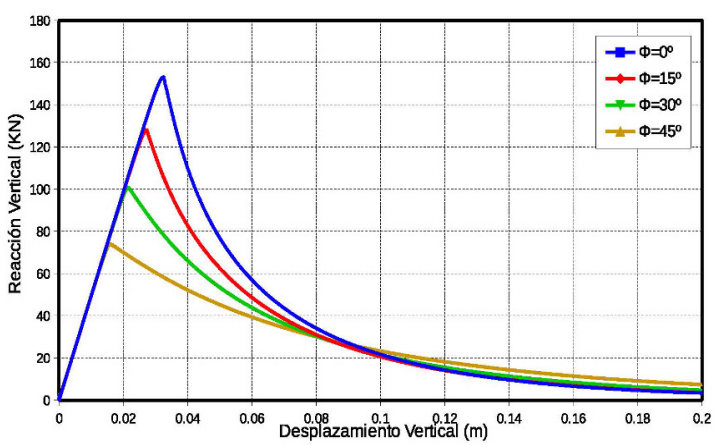

(b) DP in plane stress

Figure 22: Perforated strip. Force-displacement curves.

the DP model in plane stress, respectively. Note that, in both cases, the peak load decreases as the friction angle increases. 


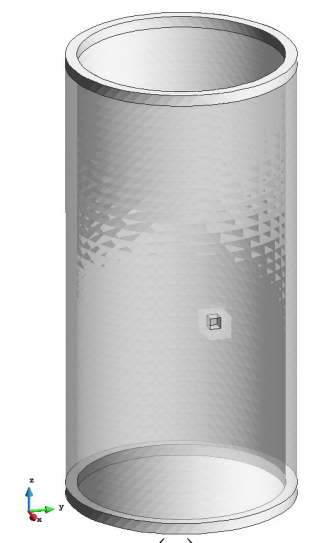

(a)

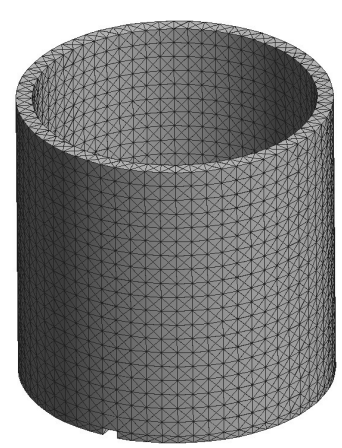

(b)

Figure 23: Perforated thin cylinder. (a) Geometry, (b) FE mesh

\subsection{Perforated thin cylinder under tension and torsion. Strain localization in $3 \mathrm{D}$}

The last problem to be studied is a perforated thin cylinder subjected to two different actions: tension and torsion along its longitudinal axis. The dimensions of the cylinder are: length $h=1.95$ $\mathrm{m}$, external radius $r=0.50 \mathrm{~m}$ and thickness $t=0.05 \mathrm{~m}$; the cylinder has a small square perforation in its midplane where stresses concentrate and one or more plastic strain localization helicoidal bands are initiated. For this geometry of the problem and for the actions considered, the stress state is plane; therefore, the localization angles are those in Table $1([10,14,51])$. Because of symmetry, only the top half of the cylinder needs to be discretized. Geometry and the structured FE mesh used, with 15357 nodes and 59880 tetrahedral MEX-FEM P1P1 elements, are shown in Figure 23.

The material properties are: density $\rho=100 \mathrm{Kg} / \mathrm{m}^{3}$, Young's modulus $E=10^{2} \mathrm{KPa}$, Poisson's ratio $\nu=0.30$, initial cohesion $c_{0}=4 \mathrm{KPa}$ and fracture energy $\mathcal{G}_{f}=5 \mathrm{~N} / \mathrm{m}$. Tension and torsion on the cylinder are induced by imposed displacements at the top plane at a velocity of $10^{-3} \mathrm{~m} / \mathrm{s}$.

Firstly, uniform longitudinal tension is studied. A von Mises plastic model is selected as yield criterion (Drucker-Prager with friction angle $\phi=0^{\circ}$ ). Figure 24 shows three different views of the shear bands formed. They are two symmetrical helicoidal bands that start at the perforation and progress towards the free end of the cylinder $\pm 45^{\circ}$ with the horizontal plane, which is the direction of the minor principal stress $([10,14,51])$. The numerical solution coincides exactly with the analytical prediction.

Secondly, uniform longitudinal torsion is studied. In this case, the Mohr-Coulomb model is used with a friction angle $\phi=45^{\circ}$. For this loading, the stress state in the walls of the cylinder is of pure shear, with principal stresses of the same value but opposite signs acting at $\pm 45^{\circ}$ with the horizontal plane. According to the analytical results in Table $1([10,14,51])$, shear band must form at $\pm 22.5^{\circ}$ with the direction of minor principal stress, that is, at $22.5^{\circ}$ and $67.5^{\circ}$ with the horizontal plane. Both alternative solutions are shown in Figures 25 and 26, respectively. In order to obtain one and the other, the torsional problem is slightly perturbed with a small longitudinal tension or compression, respectively. 


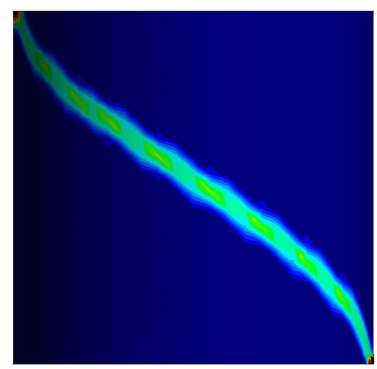

(a)

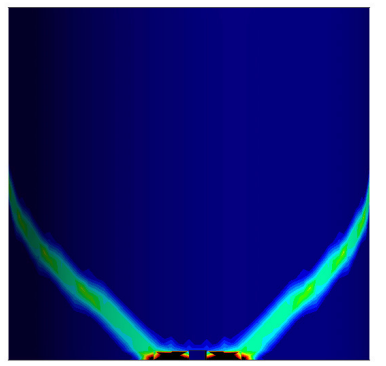

(b)

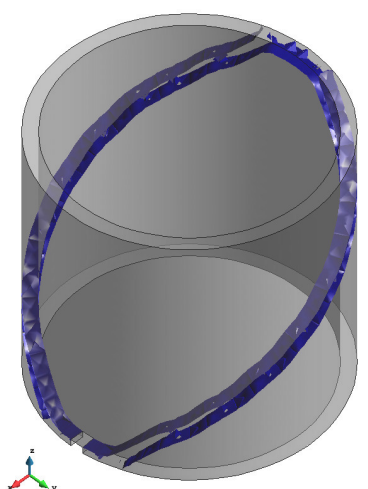

(c)

Figure 24: Perforated thin cylinder under tension. DP $\phi=0^{\circ}$. Equivalent plastic strain. Views: (a) lateral, (b) frontal and (c) isometric

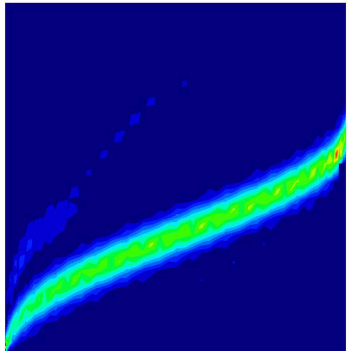

(a)

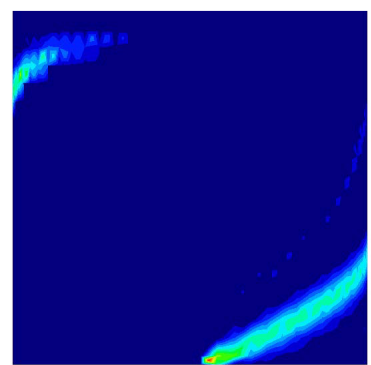

(b)

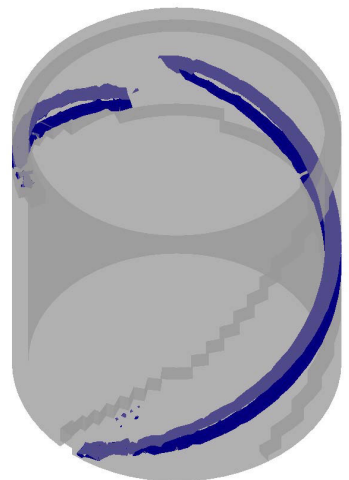

(c)

Figure 25: Perforated thin cylinder under torsion. $\mathrm{MC} \phi=45^{\circ}$. Equivalent plastic strain. Views: (a) lateral, (b) frontal and (c) isometric

Both for the cases of longitudinal tension and torsion, the analytical solutions are reproduced numerically with no spurious mesh bias. Therefore, the ability of the proposed formulation to address the problem of strain localization in elasto-plastic 3D problems is assessed. It is noteworthy that no auxiliary tracking techniques are necessary.

\section{Conclusions}

This work proposes an explicit mixed strain-displacement finite element formulation (MEX-FEM), applicable to dynamic and quasi-static problems in computational solid mechanics involving quasiincompressibility and strain localization. The formulations uses equal order interpolations and, in particular, linear/linear $P 1 P 1$ elements are demonstrated (triangles in $2 \mathrm{D}$ and tetrahedra in $3 \mathrm{D}$ ). The LBB inf-sup condition on the mixed problem is effectively circumvented using the variational multiscale method, selecting the strain and displacement subscales in the space orthogonal to the 


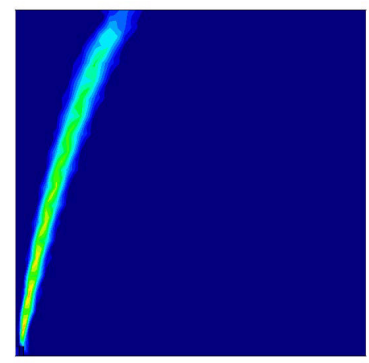

(a)

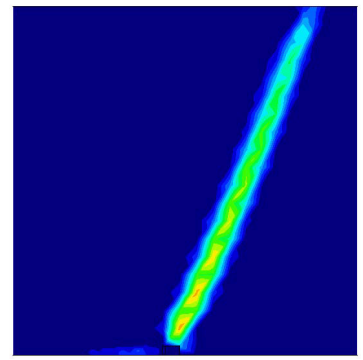

(b)

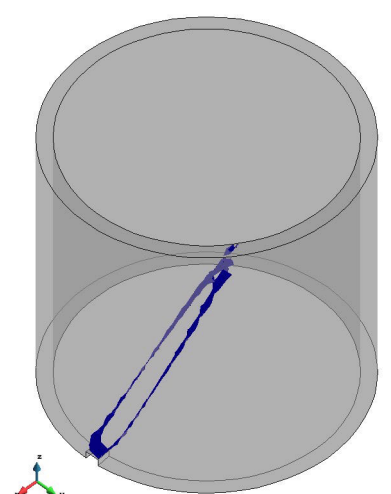

(c)

Figure 26: Perforated thin cylinder under torsion, alternative solution. MC $\phi=45^{\circ}$. Equivalent plastic strain. Views: (a) lateral, (b) frontal and (c) isometric

finite element interpolating spaces, respectively.

This provides a robust formulation which shows enhanced accuracy in the strain and stress fields when compared to the irreducible formulation and the ability to address quasi-incompressible problems when compared to previous versions on the mixed formulation that do not incorporate the displacement subscale. Additionally, an explicit implementation of the formulation, that does not require the solution of coupled full systems of equations, greatly simplifies its implementation and application.

The presented benchmark problems in 2D and 3D prove that the MEX-FEM formulation: (i) can be extended to the quasi-incompressible elastic regime, (ii) can solve problems in the compressible and quasi-incompressible plastic regime, (iii) can satisfactorily solve quasi-static material nonlinear problems involving strain localization, (iv) performs satisfactorily with directional inelastic behavior, without spurious stress locking and without the need of auxiliary discontinuity tracking procedures.

\section{Acknowledgments}

Financial support from the Spanish Ministry of Economy and Competitiveness under the project EACY - Enhanced accuracy computational and experimental framework for strain localization and failure mechanisms, ref. MAT2013-48624-C2-1-P, the Spanish Ministry of Foreign Affairs and Cooperation under the MAEC-AECID grants and the the European Commission under project NUMEXAS - Numerical Methods and Tools for Key Exascale Computing Challenges in Engineering and Applied Sciences, ref. FP7-ICT 611636, is gratefully acknowledged. 


\section{References}

[1] I. Babuska. Error-bounds for finite element method. Numerische Mathematik, 16(4):322-333, 1971.

[2] L. Benedetti, M. Cervera, and M. Chiumenti. Stress-accurate mixed fem for soil failure under shallow foundations involving strain localization in plasticity. Computers and Geotechnics, 64:962-987, 2011.

[3] D. Bigoni and T. Hueckel. Uniqueness and localization - i. associative and non-associative elastoplasticity. International Journal of Solids and Structures, 28(2):197-213, 1991.

[4] J. Bonet, A. J. Gil, C. H. Lee, M. Aguirre, and R. Ortigosa. A computational framework for polyconvex large strain elasticity. Computer Methods in Applied Mechanics and Engineering, 283:1061-1094, 2015.

[5] J. Bonet, A. J. Gil, C. H. Lee, M. Aguirre, and R. Ortigosa. A first order hyperbolic framework for large strain computational solid dynamics. part i: Total lagrangian isothermal elasticity. Computer Methods in Applied Mechanics and Engineering, 283:689-732, 2015.

[6] R. I. Borja. A finite element model for strain localization analysis of strongly discontinuous fields based on standard galerkin approximation. Computer Methods in Applied Mechanics and Engineering, 190(11-12):1529-1549, 2000.

[7] M. Cervera and M. Chiumenti. Size effect and localization in $\{$ J2 $\}$ plasticity. International Journal of Solids and Structures, 46(17):3301-3312, 2009.

[8] M. Cervera, M. Chiumenti, and C. Agelet de Saracibar. Softening, localization and stabilization: capture of discontinuous solutions in $\mathrm{j} 2$ plasticity. International Journal for Numerical and Analytical Methods in Geomechanics, 28(5):373-393, 2004.

[9] M. Cervera, M. Chiumenti, L. Benedetti, and R. Codina. Mixed stabilized finite element methods in nonlinear solid mechanics. part iii: Compressible and incompressible plasticity. Computer Methods in Applied Mechanics and Engineering, 285(0):752-775, 2015.

[10] M. Cervera, M. Chiumenti, and D. Di Capua. Benchmarking on bifurcation and localization in $\mathrm{j} 2$ plasticity for plane stress and plane strain conditions. Computer Methods in Applied Mechanics and Engineering, 241-244(0):206-224, 2012.

[11] M. Cervera, M. Chiumenti, and R. Codina. Mixed stabilized finite element methods in nonlinear solid mechanics: Part i: Formulation. Computer Methods in Applied Mechanics and Engineering, 199(37-40):2559-2570, 2010.

[12] M. Cervera, M. Chiumenti, and R. Codina. Mixed stabilized finite element methods in nonlinear solid mechanics: Part ii: Strain localization. Computer Methods in Applied Mechanics and Engineering, 199(37-40):2571-2589, 2010. 
[13] M. Cervera, M. Chiumenti, and R. Codina. Mesh objective modelling of cracks using continuous linear strain and displacement interpolations. Int. J. for Numerical Methods in Eng., $87: 32-47,2015$.

[14] M. Cervera and J.-Y. Wu. On the conformity of strong, regularized, embedded and smeared discontinuity approaches for the modeling of localized failure in solids. International Journal of Solids and Structures, 71:19-38, 2015.

[15] R. Chambon, S. Crochepeyre, and J. Desrues. Localization criteria for non-linear constitutive equations of geomaterials. Mechanics of Cohesive-frictional Materials, 5(1):61-82, 2000.

[16] M. Chiumenti, M. Cervera, and R. Codina. A mixed three-field fe formulation for stress accurate analysis including the incompressible limit. Computer Methods in Applied Mechanics and Engineering, 283(0):1095-1116, 2015.

[17] M. Chiumenti, Q. Valverde, C. Agelet de Saracibar, and M. Cervera. A stabilized formulation for incompressible elasticity using linear displacement and pressure interpolations. Computer Methods in Applied Mechanics and Engineering, 191(46):5253-5264, 2002.

[18] R. W. Clough and J. Penzien. Dynamics of structures. Earthquake Engineering \& Structural Dynamics, 1976.

[19] R. Codina. Stabilization of incompresssibility and convection through orthogonal sub-scales in finite elements methods. Comput. Meth Appl Mech Eng, 190:1579-1599, 2000.

[20] R. Codina. Stabilized finite element approximation of transient incompressible flows using orthogonal subscales. Computer Methods in Applied Mechanics and Engineering, 191(3940):4295-4321, 2002.

[21] R. Codina. Analysis of a stabilized finite element approximation of the oseen equations using orthogonal subscales. Appl. Numer. Math., 58(3):264-283, mar 2008.

[22] R. Codina and J. Blasco. A finite element method for the stokes problem allowing equal velocity-pressure interpolations. Comput. Meth Appl Mech Eng, 143:373-391, 1997.

[23] R. Codina, J. Principe, O. Guasch, and Santiago Badia. Time dependent subscales in the stabilized finite element approximation of incompressible flow problems. Computer Methods in Applied Mechanics and Engineering, 196(21-24):2413-2430, 2007.

[24] P. Dadvand, R. Rossi, M. Gil, X. Martorell, J. Cotela, E. Juanpere, S.R. Idelsohn, and E. Oñate. Migration of a generic multi-physics framework to hpc environments. Computers $\mathcal{E}_{3}$ Fluids, 80(0):301-309, 2013.

[25] P. Dadvand, R. Rossi, and E. Oñate. An object-oriented environment for developing finite element codes for multi-disciplinary applications. Archives of Computational Methods in Engineering, 17(3):253-297, 2010. 
[26] C. Agelet de Saracibar, M. Chiumenti, Q. Valverde, and M. Cervera. On the orthogonal subgrid scale pressure stabilization of finite deformation $\{\mathrm{J} 2\}$ plasticity. Computer Methods in Applied Mechanics and Engineering, 195(9-12):1224-1251, 2006.

[27] F. Brezzi, M. Fortin, D. Marini. Mixed finite element methods. Springer, 1991.

[28] GiD. The personal pre and post processor. http://gid.cimne.upc.es. 2009.

[29] A. J. Gil, C. H. Lee, J. Bonet, and M. Aguirre. Stabilised petrov-galerkin formulation for linear tetrahedral elements in compressible, nearly incompressible and truly incompressible fast dynamics. Computer Methods in Applied Mechanics and Engineering, 276(0):659-690, 2014.

[30] T. J. R. Hughes. Generalization of selective integration procedures to anisotropic and nonlinear media. International Journal for Numerical Methods in Engineering, 15(9):1413-1418, 1980.

[31] T. J. R. Hughes, G. Scovazzi, and L. P. Franca. Multiscale and Stabilized Methods. John Wiley \& Sons, Ltd, 2004.

[32] T. J.R. Hughes, G. R. Feijóo, L. Mazzei, and J.-B. Quincy. The variational multiscale method-a paradigm for computational mechanics. Computer Methods in Applied Mechanics and Engineering, 166(1-2):3-24, 1998. Advances in Stabilized Methods in Computational Mechanics.

[33] T.J.R. Hughes. Multiscale phenomena: Green's functions, the dirichlet-to-neumann formulation, subgrid scale models, bubbles and the origins of stabilized methods. Computer Methods in Applied Mechanics and Engineering, 127(1-4):387-401, 1995.

[34] M. Iordache and K. Willam. Localized failure analysis in elastoplastic cosserat continua. Computer Methods in Applied Mechanics and Engineering, 151(3-4):559-586, 1998. Containing papers presented at the Symposium on Advances in Computational Mechanics.

[35] N.M. Lafontaine, R. Rossi, M. Cervera, and M. Chiumenti. Explicit mixed strain-displacement finite element for dynamic geometrically non-linear solid mechanics. Computational Mechanics, pages $1-17,2015$.

[36] C. H. Lee, A. J. Gil, and J. Bonet. Development of a stabilised petrov-galerkin formulation for conservation laws in lagrangian fast solid dynamics. Computer Methods in Applied Mechanics and Engineering, 268:40-64, 2014.

[37] Y. Leroy and M. Ortiz. Finite element analysis of transient strain localization phenomena in frictional solids. International Journal for Numerical and Analytical Methods in Geomechanics, 14(2):93-124, 1990.

[38] D. S. Malkus and T.J.R. Hughes. Mixed finite element methods - reduced and selective integration techniques: A unification of concepts. Computer Methods in Applied Mechanics and Engineering, 15(1):63-81, 1978. 
[39] J.C. Nagtegaal, D.M. Park, and J.R. Rice. On numerically accurate finite element solutions in the fully plastic range. Computer Methods in Applied Mechanics and Engineering, 15(4):153$177,1974$.

[40] E.d.S. Neto and D.R.J. Peric. Computational Methods for Plasticity. Wiley, 2008.

[41] E. Oñate, J. Rojek, R. L. Taylor, and O. C. Zienkiewicz. Finite calculus formulation for incompressible solids using linear triangles and tetrahedra. International Journal for Numerical Methods in Engineering, 59(11):1473-1500, 2004.

[42] E. Oñate, A. Valls, and J. GarcÃya. Fic/fem formulation with matrix stabilizing terms for incompressible flows at low and high reynolds numbers. Computational Mechanics, 38(45):440-455, 2006.

[43] N. Saabye Ottosen and K. Runesson. Properties of discontinuous bifurcation solutions in elasto-plasticity. International Journal of Solids and Structures, 27(4):401-421, 1991.

[44] J.W. Rudnicki and J.R. Rice. Conditions for the localization of deformation in pressuresensitive dilatant materials. Journal of the Mechanics and Physics of Solids, 23(6):371-394, 1975 .

[45] K. Runesson, N. Saabye Ottosen, and P. Dunja. Discontinuous bifurcations of elastic-plastic solutions at plane stress and plane strain. International Journal of Plasticity, 7(1-2):99-121, 1991.

[46] J. C. Simo and T. J. R. Hughes. Computational Inelasticity. Springer-Verlag, New-York, 1998.

[47] J.C. Simo, R.L. Taylor, and K.S. Pister. Variational and projection methods for the volume constraint in finite deformation elasto-plasticity. Computer Methods in Applied Mechanics and Engineering, 51(1-3):177-208, 1985.

[48] P. Steinmann and K. Willam. Finite element analysis of elastoplastic discontinuities. Journal of Engineering Mechanics, 120(11):2428-2442, 1994.

[49] R. L. Taylor. A mixed-enhanced formulation tetrahedral finite elements. International Journal for Numerical Methods in Engineering, 47(1-3):205-227, 2000.

[50] Q. Valverde, M. Chiumenti, M. Cervera, and C. Agelet de Saracibar. Formulación estabilizada de elementos finitos triangulares y tetraédricos para problemas de incompresibilidad en pequeñas deformaciones. Revista Internacional de Métodos Numéricos para Cálculo y Diseño en Ingeniería, 21(4), 2005.

[51] J.-Y. Wu and M. Cervera. On the equivalence between traction- and stress-based approaches for the modeling of localized failure in solids. Journal of the Mechanics and Physics of Solids, 82:137-163, 2015 . 